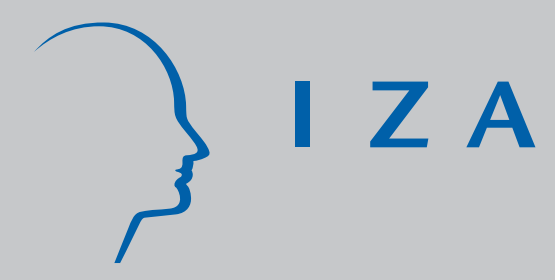

IZA DP No. 949

Minimum Wage Effects on Labor Market

Outcomes under Search with Bargaining

Christopher J. Flinn

December 2003 


\title{
Minimum Wage Effects on Labor Market Outcomes under Search with Bargaining
}

\author{
Christopher J. Flinn \\ New York University and IZA Bonn
}

Discussion Paper No. 949

December 2003

\author{
IZA \\ P.O. Box 7240 \\ D-53072 Bonn \\ Germany \\ Tel.: +49-228-3894-0 \\ Fax: +49-228-3894-210 \\ Email: iza@iza.org
}

This Discussion Paper is issued within the framework of IZA's research area Evaluation of Labor Market Policies and Projects. Any opinions expressed here are those of the author(s) and not those of the institute. Research disseminated by IZA may include views on policy, but the institute itself takes no institutional policy positions.

The Institute for the Study of Labor (IZA) in Bonn is a local and virtual international research center and a place of communication between science, politics and business. IZA is an independent, nonprofit limited liability company (Gesellschaft mit beschränkter Haftung) supported by Deutsche Post World Net. The center is associated with the University of Bonn and offers a stimulating research environment through its research networks, research support, and visitors and doctoral programs. IZA engages in (i) original and internationally competitive research in all fields of labor economics, (ii) development of policy concepts, and (iii) dissemination of research results and concepts to the interested public. The current research program deals with (1) mobility and flexibility of labor, (2) internationalization of labor markets, (3) welfare state and labor market, (4) labor markets in transition countries, (5) the future of labor, (6) evaluation of labor market policies and projects and (7) general labor economics.

IZA Discussion Papers often represent preliminary work and are circulated to encourage discussion. Citation of such a paper should account for its provisional character. A revised version may be available on the IZA website (www.iza.org) or directly from the author. 


\title{
ABSTRACT
}

\section{Minimum Wage Effects on Labor Market Outcomes under Search with Bargaining*}

\begin{abstract}
Building upon a continuous-time model of search with Nash bargaining in a stationary environment, we analyze the effect of changes in minimum wages on labor market outcomes and welfare. While minimum wage increases invariably lead to employment losses in our model, they may be welfare-improving to labor market participants using any one of a number of welfare criteria. A key determinant of the welfare impact of a minimum wage increase is the Nash bargaining power parameter. We discuss identification of this model using Current Population Survey data on accepted wages and unemployment durations, and demonstrate that key parameters are not identified when the distribution of match values belongs to a location-scale family. By incorporating a limited amount of information from the demand side of the market, we are able to obtain credible and precise estimates of all primitive parameters, including bargaining power. Direct estimates of the welfare impact of the minimum wage increase from $\$ 4.25$ to $\$ 4.75$ in 1996 provide limited evidence of a small improvement. Using estimates of the primitive parameters we show that more substantial welfare gains for labor market participants could have been obtained by doubling the minimum wage rate in 1996, though at the cost of a perhaps unacceptably high unemployment rate.
\end{abstract}

JEL Classification: C34, C51, J41, J38

Keywords: minimum wages, matching models, Nash bargaining

Christopher J. Flinn

Department of Economics

New York University

269 Mercer Street

New York, NY 10003

USA

Email: christopher.flinn@nyu.edu

\footnotetext{
* This research is partially supported by the W.E. Upjohn Institute for Employment Research and the C.V. Starr Center for Applied Economics at NYU. I have received many useful comments from participants in seminars held at North Carolina, Johns Hopkins, Columbia, the New York Fed, NYU, LSE, Yale, Bocconi, and the BLS; and in conferences, including the Econometric Society Meetings (New York, 1999), the Institute for Research on Poverty Summer Workshop (Madison, 1999), the Society for Economic Dynamics (Sardegna, 1999), and the European Econometric Society Meetings (Santiago de Compostela, 1999). I am especially grateful to Jim Albrecht, Matt Dey, Luca Flabbi, Sarah Hamersma, Winfried Koeniger, Bernard Salanié, and Holger Sieg for helpful comments and suggestions. I remain solely responsible for all errors, omissions, and interpretations.
} 


\section{Introduction}

Determining the equilibrium effects of minimum wage changes on labor market outcomes is a challenging modeling and estimation problem; arriving at policy recommendations is a task even more daunting. Faced with the inherent difficulties of modeling equilibrium labor market events given the limited amount of data to which researchers have access, much recent research has been performed outside of an explicit behavioral framework, with researchers pursuing the more limited objective of carefully describing the observed effects of recent minimum wage changes using quasiexperimental methods [see Card and Krueger (1995), hereafter CK, for a summary of these studies and a comprehensive, critical survey of most of the previous research done in this area]. In our view, these recent studies have been particularly useful in indicating that the "textbook" competitive model of the labor market, which has been used as an interpretive framework for the bulk of empirical studies using aggregated time series data, may have serious deficiencies in accounting for minimum wage effects on labor market outcomes when confronted with disaggregated data.

While the quasi-experimental results have raised a number of interesting challenges to orthodox theory, few cogent models have been advanced that are consistent with the results that have been found. Some of the explanations for these empirical findings (e.g., lack of significant employment losses, impacts on the wage distribution above the minimum) do not seem to be testable given our current data resources. In fact, it appears difficult to operationalize many of the explanations proposed even for the more modest purpose of empirical implementation.

A fully-elaborated, competitive equilibrium model of the effects of minimum wages presumably would require a system of labor demand functions for various observationally-differentiated classes of labor and capital in addition to a well-specified system of labor supply functions. Using individuallevel data on individuals at a point in time or from a short panel, the requisite information on the demand-side of the market would be missing, so that to estimate an equilibrium model in this case requires one to make a number of stringent assumptions concerning the behavior of firms [see Eckstein and Wolpin (1990), van den Berg and Ridder (1993), and Flinn (1997) for examples of the estimation of equilibrium labor market models using only individual-level data on workers]. Even after making such assumptions, it may be the case that important demand-side parameters will be unidentified, thus making it problematic to conduct policy experiments. Therefore this approach has some severe limitations from the point of view of implementation, and additionally may not be able to generate certain key features of the observed wage distribution, such as the probability mass at the minimum wage.

It has long been recognized that for the imposition of minimum wages to have a beneficial effect on the welfare of all labor market participants on the supply side of the market, firms must have some degree of monopsony power [see for example Manning (1995)]. Search frictions and the existence of match-specific capital are capable of providing this. The existence of both seems in accord with common sense and empirical findings [on the existence of match-specific capital see Miller (1984) and Flinn (1986), for example]. Below we formulate a model which includes matchspecific capital, job search, and worker-firm bargaining over match-specific rents, that is capable of accounting for most of the empirical observations cited by CK in their survey of minimum wage research based on individual level data (1995, ch. 7).

To illustrate some of the features of wage distributions (and unemployment phenomena) CK point to, consider Figure 1. This figure contains plots of hourly wage distributions and the distributions of on-going spells of unemployed search for individuals 16-24 years of age in the Outgoing Rotation Groups (ORG) of the Current Population Survey (CPS) in September and October 1996. 
The federal minimum wage was officially changed from $\$ 4.25$ to $\$ 4.75$ on September 30,1996 . The hourly wage distributions that appear in panels 1.a and 1.c exhibit a number of common features. In both cases, there are hourly wage rates below the mandated minimum wage. In addition, both distributions exhibit a large amount of probability mass concentrated on relatively few points. In September the wage value $\$ 4.25$ has the fourth largest probability mass and the same is true in October with respect to the value $\$ 4.75$. In terms of an overall impression of these distributions, if one were to smooth the probability mass away from the spikes (except at the minimum wage rates) and ignore the small number of wage observations below the minimum wages the data seem consistent with draws from a truncated log normal distribution. In the sequel we focus on the lognormal distribution because of its predominance in studies of wage determination.

The model developed below will generate at most one mass point in the wage distribution, at the prevailing minimum wage, so it may be worthwhile to comment on the appearance of so many mass points in the empirical distribution. In the descriptive statistics presented in Table 1, we see that the proportion of employed individuals paid $\$ 4.25$ in September was 0.059 while in October the proportion paid $\$ 4.75$ was 0.070 . The largest probability mass in both months is associated with the value $\$ 5.00$, which is the wage reported for between 13 and 14 percent of the employed youth. Other especially large mass points are associated with the values of $\$ 6.00, \$ 7.00$, and $\$ 8.00$ in both months. We believe that it is reasonable to suppose that these values are simply "focal points" for respondents, who in many cases are not even the individual whose wage is being reported. ${ }^{1}$ The fact that the minimum wage was not set at values that could be characterized as natural focal points may lend some strength to our claim that the mass point at the minimum wage is the only "real" one in these empirical wage distributions.

Finally, we wish to record a few observations about unemployment experiences of the sample members. Using standard definitions of the unemployment state, we find that there was a small increase in the unemployment rate (Table 1) in October 1996, an observation that is consistent with the model developed below. Unlike much current thinking on minimum wage impacts on the distribution of welfare, under our model this unemployment increase does not necessarily imply a deleterious effect of the minimum wage increase of September 301996 on all, or even any, population members. We note that the steady state model we analyze below carries the implication that the duration of on-going job search reported by unemployed CPS members should follow a negative exponential distribution. While there are clearly a number of mass points in these data, which are most probably produced by reporting error, neither distribution is grossly inconsistent with the negative exponential. ${ }^{2}$

The model is a logical descendent of the econometric model of search of Flinn and Heckman (1982) - hereafter referred to as FH - and the econometric model of minimum wage effects on the distribution of wages and the probability of employment estimated by Meyer and Wise (1983a,b) - hereafter referred to as MW. FH formulated an equilibrium continuous-time search model in which searchers encountered potential employers according to a Poisson process; upon meeting, the potential value of the contact was determined by a draw from a fixed distribution $G$. In their analysis the match value was arbitrarily assumed to be divided evenly between workers and firms. The setup of the basic model proposed here is similar, with the important difference that rents are

\footnotetext{
${ }^{1}$ We cannot substantiate this claim since we do not have access to the payroll records of the subjects' employers.

${ }^{2}$ As we shall show formally below, the on-going unemployment distribution in October should not exactly belong to the negative exponential family under our model. This is due to the fact that it is not a steady state distribution because of the (then) recent change in the minimum wage.
} 
divided using an explicit Nash-bargaining criteria. The bargaining power parameter which appears in this formulation is of key importance in determining the welfare consequences [on labor market participants] of imposing, or increasing, a minimum wage.

It is worth elaborating on the role of the bargaining power parameter in this research. As we have noted, this parameter did not appear in the formulation of $\mathrm{FH}$ - for one reason because it is a more difficult parameter to interpret than are the others in their model. We think of it as comprising a sort of summary statistic of the labor market "position" of a particular group. For example, the match value distribution for low-skilled workers may be stochastically dominated by the match value distribution for high-skilled workers, but in addition low-skilled workers may be at a disadvantage due to their having little bargaining power. Their low bargaining power may derive from there being many substitutes for them in the production process, or to the relative number of low-skilled workers to the number of positions for this type of worker. In this sense, the parameter cannot be really thought of as "primitive" since significant policy changes - such as a doubling of the minimum wage - may result in participation effects or substitution responses by firms which change the labor market "position" of the group, and hence change the bargaining power parameter. Thus comparative statics exercises and policy experiments performed with the estimates obtained from this model will only be valid locally, that is, for small changes in policy variables. This limitation is not too disturbing, since it probably applies to all empirical research which has been conducted in this area.

MW estimated a model of minimum wage effects using individual-level data which allowed them to infer what the wage distribution and employment level would have been in the absence of a minimum wage. Their model was both original and suggestive. While it has been criticized by a number of researchers [e.g., CK (pp.232-236) and Dickens et al. (1997)], primarily for relying on functional form assumptions for identification and for choosing a parameterization that rules out the possibility of employment increases in response to a minimum wage increase, it remains one of the better econometric attempts to identify minimum wage effects using individual-level data in the literature [another example is Heckman and Sedlacek (1981)]. From our perspective, the main weakness of their model is the arbitrary specification of the manner in which a minimum wage "distorts" the preexisting distribution wage distribution. In our model, optimizing behavior by searchers and firms determines the nature of this "distortion," and it is roughly consistent both with the MW specification and with the empirical evidence cited in CK.

In this research we attempt to integrate theory and measurement in analyzing minimum wage effects on the labor market. For example, while there are a number of papers in the literature which consider the possibility of welfare-enhancing minimum wage rates [e.g., Drazen (1986), Lang (1987), Rebitzer and Taylor (1996), Swinnerton (1996)], the frameworks in which the models are set tend to be relatively abstract and the models themselves are typically unestimable. In this research, the same model which is used to define conditions under which minimum wages can be welfare-improving for the supply side of the market is estimated using individual-level data. With the parameter estimates obtained, we are able to check whether actual minimum wage changes have been welfare-improving for searchers and employees, as well as to make some informed speculation as to the optimal level of the minimum wage [once again, considering only searchers and employees] under certain well-defined welfare criteria.

The plan of the paper is as follows. In Section 2 we develop the bargaining model in a continuoustime search environment both in the absence and in the presence of a binding minimum wage. Section 3 contains a discussion of the welfare effects of the imposition of a minimum wage, and 
presents two summary measures of these effects. We also explore in some detail the effect of changes in minimum wage laws on labor market outcomes such as the wage distribution and employment rate, as well as on the profit rate of firms. In Section 4, we begin by developing an econometric framework which is used to estimate the model. Issues of model identification are considered in some depth. In Section 5 we present results from estimating various specifications of the bargaining model using CPS-ORG data from September and October 1996. Section 6 employs model estimates to determine optimal levels of minimum wages under a variety of welfare criteria. A conclusion is offered in Section 7.

\section{$2 \quad$ Labor Market Search with Bargaining}

In this section we describe the behavioral model of labor market search with matching and bargaining. The model is formulated in continuous time and assumes stationarity of the labor market environment. In the first subsection we derive the decision rules for terminating search and for dividing the match value between worker and firm in the absence of minimum wages. In the following subsection we describe the manner in which minimum wages affect search behavior and the division of the match value.

Throughout we assume that there exists an invariant, technologically-determined distribution of worker-firm productivity levels which is given by $G(\theta)$. When a potential employee and a firm meet, which happens at rate $\lambda$, the productive value of the match $(\theta)$ is immediately observed by both the applicant and the firm. At this point a division of the match value is proposed using a Nash bargaining framework. The searcher's instantaneous discount rate is given by $\rho>0$. The rate of (exogenous) termination of employment contracts is $\eta \geq 0$. While unemployed individuals search, their instantaneous utility is given by $b$, which can assume positive or negative values. For simplicity, we assume that employed individuals do not receive alternative offers of employment, i.e., there is no on-the-job search. It is straightforward to adapt the current framework to that case, however. ${ }^{3}$

\subsection{Labor Market Decisions without Minimum Wages}

We assume that the only factor of production is labor, and that total output of the firm is simply the sum of the productivity levels of all of its employees. Then if the firm "passes" on the applicant - that is, does not make an employment offer - its "disagreement" outcome is 0 [it earns no revenue but makes no wage payment]. The applicant's disagreement value is the value of continued search, which we denote $V_{n}$. For any given value of $V_{n}$ there exists a corresponding critical "match" value $\theta^{*}=\rho V_{n}$ ( $\rho$ is the instantaneous discount rate), which has the property that all matches with values at least as great as $\theta^{*}$ will result in employment while all those matches of lower value will not. For any $\theta \geq \theta^{*}$, the wage offer is given by

$$
w\left(\theta, V_{n}\right)=\arg \max _{w}\left[V_{e}(w)-V_{n}\right]^{\alpha}\left[\frac{\theta-w}{\rho+\eta}\right]^{1-\alpha},
$$

where without loss of generality it has been assumed that the firm shares the employee's effective rate of discount, $\rho+\eta$.

\footnotetext{
${ }^{3}$ Since the CPS data used in the empirical analysis are essentially cross-sectional, it is virtually impossible to construct the kind of event history data required to estimate a model with sophisticated turnover phenomena.
} 
The value of employment at a wage of $w$ is easily determined. Consider an infinitesimally small period of time $\varepsilon$. Over this "period," either the individual will continue to be employed at wage $w$ or will lose their job, which occurs at rate $\eta$. Then

$$
V_{e}(w)=(1+\rho \varepsilon)^{-1}\left\{w \varepsilon+\eta \varepsilon V_{n}+(1-\eta \varepsilon) V_{e}(w)+o(\varepsilon)\right\}
$$

where the term $(1+\rho \varepsilon)^{-1}$ is an "infinitesimal" discount factor associated with the small interval $\varepsilon, \eta \varepsilon$ is the approximate probability of being terminated from one's current employment by the end of $\varepsilon$, and $o(\varepsilon)$ is a term which has the property that $\lim _{\varepsilon \rightarrow 0}(o(\varepsilon) / \varepsilon)=0$. Note that the first term on the right hand side of [2] is the value of the wage payment over the interval, which is the total payment $w \varepsilon$ multiplied by the "instantaneous" discount factor [think of the payment as being received at the end of the interval $\varepsilon]$. After collecting terms and taking the limit of $[2]$ as $\varepsilon \rightarrow 0$, we have

$$
V_{e}(w)=\frac{w+\eta V_{n}}{\rho+\eta}
$$

We now substitute [3] into [1] so as to simplify the problem as follows:

$$
\begin{aligned}
V_{e}(w)-V_{n} & =\frac{w+\eta V_{n}}{\rho+\eta}-V_{n} \\
& =\frac{w-\rho V_{n}}{\rho+\eta},
\end{aligned}
$$

so that we get the well-known expression

$$
\begin{aligned}
w\left(\theta, V_{n}\right) & =\arg \max _{w}\left[w-\rho V_{n}\right]^{\alpha}[\theta-w]^{1-\alpha} \\
& =\alpha \theta+(1-\alpha) \rho V_{n} .
\end{aligned}
$$

We can now move onto computing the value of nonemployment. Using the same setup as above for defining the value of employment, we begin with the $\varepsilon$-period formulation

$$
\begin{aligned}
& V_{n}=(1+\rho \varepsilon)^{-1}\left\{b \varepsilon+\lambda \varepsilon \int \max \left[V_{n}, V_{e}\left(w\left(\theta, V_{n}\right)\right)\right] d G(\theta)\right. \\
& \left.+(1-\lambda \varepsilon) V_{n}+o(\varepsilon)\right\}
\end{aligned}
$$

where $b \varepsilon$ is the utility associated with unemployment over the interval $\varepsilon$ and $\lambda \varepsilon$ is the approximate probability of encountering one potential employer within the period of duration $\varepsilon$. Rearranging and taking limits, we have

$$
\rho V_{n}=b+\lambda \int_{\rho V_{n}}\left[V_{e}\left(w\left(\theta, V_{n}\right)\right)-V_{n}\right] d G(\theta) .
$$

Since

$$
\begin{aligned}
V_{e}\left(w\left(\theta, V_{n}\right)\right) & =\frac{\alpha \theta+(1-\alpha) \rho V_{n}+\eta V_{n}}{\rho+\eta} \\
& =\frac{\alpha \theta-\alpha \rho V_{n}}{\rho+\eta}+V_{n},
\end{aligned}
$$

we have

$$
V_{e}\left(w\left(\theta, V_{n}\right)\right)-V_{n}=\frac{\alpha \theta-\alpha \rho V_{n}}{\rho+\eta}
$$


Then the final (implicit) expression for the value of search is

$$
\rho V_{n}=b+\frac{\alpha \lambda}{\rho+\eta} \int_{\rho V_{n}}\left[\theta-\rho V_{n}\right] d G(\theta) .
$$

Note that this expression is identical to the expression for the reservation value in a model with no bargaining when $\theta$ is the payment to the individual except for the presence of the factor $\alpha$. This is not unexpected, since when $\alpha=1$, the entire match value is transferred to the worker, and thus search over $\theta$ is the same as search over $w$.

Now we can summarize the important properties of the model. The critical "match" value $\theta^{*}$ is equal to $\rho V_{n}$, which is defined by [4]. Since at this match value the wage payment is equal to $w^{*} \equiv w\left(\theta^{*}, V_{n}\right)=\alpha \theta^{*}+(1-\alpha) \theta^{*}=\theta^{*}$, the reservation wage is identical to the reservation match value. The probability that a random encounter generates an acceptable match is given by $\tilde{G}\left(\theta^{*}\right)$, where $\tilde{G}$ denotes the survivor function, $1-G$. The rate of leaving unemployment is $\lambda \tilde{G}\left(\theta^{*}\right)$. As we can see from [4], since $\theta^{*}$ is an increasing function of $\alpha$, rates of unemployment are higher when searchers have more bargaining power. Since supply-side welfare, no matter how measured, is a non-decreasing function of $\alpha$, it is clear that in this type of model high unemployment rates are not necessarily associated with low welfare values for employees and unemployed searchers.

The observed wage density is a simple mapping from the matching density. Since

$$
\begin{aligned}
w\left(\theta, V_{n}\right) & =\alpha \theta+(1-\alpha) \theta^{*} \\
\Rightarrow \tilde{\theta}\left(w, V_{n}\right) & =\frac{w-(1-\alpha) \theta^{*}}{\alpha},
\end{aligned}
$$

then the density function of observed wages is given by

$$
h(w)=\left\{\begin{array}{ll}
\frac{\alpha^{-1} g\left(\tilde{\theta}\left(w, V_{n}\right)\right)}{\tilde{G}\left(\theta^{*}\right)} & w \geq \theta^{*} \\
0 & w<\theta^{*}
\end{array} .\right.
$$

It will be useful to carry an example through the first part of the paper, particularly in order to emphasize the key role the bargaining power parameter $\alpha$ plays in determining the substantive implications of the model. In our example labor market we set the rate of arrival of offers $(\lambda)$ to the value .5 (so that job contacts occur every 2 "periods" on average), the rate of job dissolutions $(\eta)$ is set to .02 (so that the average length of a job is 50 periods), $\rho$ is set to .01, and the instantaneous return from search $(b)$ is set to -1 . The firm-searcher matching distribution is assumed to be uniform with support $[0,10]$. We will compute the equilibrium wage distribution for $\alpha=.3$ and $\alpha=.6$.

Figure 2.a plots the uniform p.d.f. which represents $g(\theta)$ in this case. Figure 2.b plots the mapping from draws of $\theta$ into wage offers under the alternative values of $\alpha$, that is $w_{\alpha}\left(\theta, V_{n}(\alpha)\right)=$ $\alpha \theta+(1-\alpha) \rho V_{n}(\alpha)$. Note that $\alpha$ affects the equilibrium mapping both directly through the slope and indirectly through the disagreement point $\rho V_{n}(\alpha)$. Figures 2.c-d plot the equilibrium wage p.d.f.s for the two $\alpha$ values. Increasing $\alpha$ in the uniform case simply results in increases in the lower and upper bound of the support of the equilibrium wage distribution, which is itself uniform.

\subsection{Labor Market Decisions in the Presence of Minimum Wages}

Now consider the case in which the interactions between applicants and firms are constrained by the presence of a minimum wage. The minimum wage, $m$, is set by the government and is assumed 
to apply to all potential matches. We assume that the only compensation provided by the firm is the wage. Thus there are no other forms of compensation the firm can adjust so as to "undo" the minimum wage payment requirement.

We impose the minimum wage in the framework established in the previous section. As should be clear, any $m \leq \theta^{*}$ has no effect on the behavior of applicants or firms and thus would be a meaningless constraint. Thus we consider only the effects of the imposition of an $m>\theta^{*}$.

Recall that the expected value of the match from the point of view of the firm is proportional to $(\theta-w)$. Firms cannot earn positive profits on matches which have a value less than $m$. Since $m>\theta^{*}$, an immediate implication of the imposition of the minimum wage is that fewer contacts will result in jobs - the standard employment effect.

In terms of wage payments, the minimum wage acts solely as a side constraint on the Nash bargaining problem. Formally, the revised problem is given by

$$
w\left(\theta, V_{n}\right)=\arg \max _{w \geq m}\left[V_{e}(w)-V_{n}\right]^{\alpha}\left[\frac{\theta-w}{\rho+\eta}\right]^{1-\alpha},
$$

where the only difference from [1] is the restriction $w \geq m$. The effect on the solution is relatively intuitive. Under the "constrained" Nash bargaining problem, there will exist a value of search which we denote $V_{n}(m)$ [this value is not equal to the $V_{n}$ defined in the previous subsection - it will be defined below]. If we ignore the minimum wage constraint in determining the wage payment given a match value of $\theta$ and the search value $V_{n}(m)$, we get

$$
w\left(\theta, V_{n}(m)\right)=\alpha \theta+(1-\alpha) \rho V_{n}(m) .
$$

Under this division of the match value, the worker would receive a wage of $m$ when $\theta=\hat{\theta}$, where

$$
\hat{\theta}\left(m, V_{n}(m)\right)=\frac{m-(1-\alpha) \rho V_{n}(m)}{\alpha} .
$$

Then if $\hat{\theta} \leq m$, all "feasible" matches would generate wage offers at least as large as $m$. When $\hat{\theta}>m$, this is not the case. When $\theta$ belongs to the set $[m, \hat{\theta})$, the offer according to [5] is less than $m$. However, when confronted with the choice of giving some of its surplus to the worker versus a return of 0 , the firm pays the wage of $m$ for all $\theta \in[m, \hat{\theta})$. Wages for acceptable $\theta$ outside of this set are determined according to [5].

We can now consider the individual's search problem given this wage offer function. Using the $\varepsilon$ look ahead formulation,

$$
\begin{aligned}
V_{n}(m)= & (1+\rho \varepsilon)^{-1}\left\{b \varepsilon+\lambda \varepsilon\left[\int_{m}^{\hat{\theta}\left(m, V_{n}(m)\right)}\left[\frac{m+\eta V_{n}(m)}{\rho+\eta}-V_{n}(m)\right] d G(\theta)\right.\right. \\
& \left.+\int_{\hat{\theta}\left(m, V_{n}(m)\right)}\left[\frac{\alpha \theta+(1-\alpha) \rho V_{n}(m)+\eta V_{n}(m)}{\rho+\eta}-V_{n}(m)\right] d G(\theta)+V_{n}(m)\right] \\
& \left.+(1-\lambda \varepsilon) V_{n}(m)+o(\varepsilon)\right\} . \\
= & (1+\rho \varepsilon)^{-1}\left\{b \varepsilon+\lambda \varepsilon\left[\int_{m}^{\hat{\theta}\left(m, V_{n}(m)\right)}\left[\frac{m-\rho V_{n}(m)}{\rho+\eta}\right] d G(\theta)\right.\right. \\
& \left.\left.+\int_{\hat{\theta}\left(m, V_{n}(m)\right)}\left[\frac{\alpha\left(\theta+\rho V_{n}(m)\right)}{\rho+\eta}\right] d G(\theta)+V_{n}(m)\right]+(1-\lambda \varepsilon) V_{n}(m)+o(\varepsilon)\right\} .
\end{aligned}
$$


Taking limits after collecting terms, we have

$$
\begin{aligned}
\rho V_{n}(m)= & b+\frac{\lambda}{\rho+\eta}\left\{\int_{m}^{\hat{\theta}\left(m, V_{n}(m)\right)}\left[m-\rho V_{n}(m)\right] d G(\theta)\right. \\
& \left.+\alpha \int_{\hat{\theta}\left(m, V_{n}(m)\right)}\left[\theta-\rho V_{n}(m)\right] d G(\theta)\right\} .
\end{aligned}
$$

We shall often refer to the value $\rho V_{n}(m)$ as the "implicit" reservation wage. Unlike the situation in which a binding minimum wage is not present, this value is not the minimal acceptable wage and match value. The acceptable wage/match value is rather the imposed minimum value $m$. Nonetheless, the value $\rho V_{n}(m)$ is of critical importance in determining equilibrium wages and the welfare impacts of minimum wage changes.

Conditional on the value of a binding minimum wage $m$, the equilibrium wage distribution is described by

$$
p(w ; m)=\left\{\begin{array}{cc}
\frac{\alpha^{-1} g\left(\tilde{\theta}\left(w, V_{n}(m)\right)\right)}{\tilde{G}(m)} & w>m \\
\frac{\tilde{G}(m)-\tilde{G}\left(\hat{\theta}\left(m, V_{n}(m)\right)\right.}{\tilde{G}(m)} & w=m \\
0 & w<m
\end{array}\right.
$$

The minimum wage side constraint produces an equilibrium wage distribution which has a mass point at $m$ and has wages being continuously distributed on the interval $(m, \infty){ }^{4}$

Let us reconsider our uniform example after a minimum wage of 7 has been imposed; since the distribution now has a mass point, it is necessary to plot the c.d.f. as opposed to the p.d.f. Figure 3.a plots the c.d.f. of the matching distribution. Figure 3.b contains the equilibrium wage offer mapping from $\theta$ to $w$ when $m=7$. Note that for the case of $\alpha=.3$ the equilibrium wage function maps all values of $\theta \geq 7$ into a wage offer of $w=7$. At least when the distribution $G$ has bounded support, this demonstrates that the imposition of a minimum wage can result in a degenerate wage offer distribution at the minimum, as we see in Figure 3.c. In the case of $\alpha=.6$ (Figure 3.d), the equilibrium wage distribution has a substantial mass point at 7 , with a relatively narrow range of wages above it.

\section{The Effects of Minimum Wage Changes}

This section is divided into four parts. In the first we propose three different welfare criteria under which minimum wage impacts can be evaluated. In this subsection we only consider welfare impacts on the supply side of the market. We then go on to consider the employment effects of minimum wages in the context of our model, and follow this with a discussion of the manner in which the wage distribution shifts. The section concludes with a discussion of the impact of minimum wages on employer welfare. While our main focus throughout is be on the welfare of individuals on the supply side of the market, we include this subsection for reasons of symmetry and because information on firm performance will be seen to be critical for the econometric identification of the model.

\footnotetext{
$R_{+}$.

${ }^{4}$ This statement is predicated on $\theta$ being a continuously distributed random variable with unbounded support on
} 


\subsection{Welfare Criteria}

We present a discussion of three welfare measures that pertain solely to the supply side of the market. Since most minimum wage legislation that has been proposed has cited concern for the welfare of individuals rather than firms, these minimum wage criteria may be a particularly interesting focus of applied research. Moreover, we should note that by the structure of our bargaining model, all firms will be able to remain solvent no matter what the level of the minimum wage, which is not of course to say that they are indifferent with respect to its level. We will discuss firm welfare in more detail below.

\subsubsection{Value of Unemployed Search $(W C 1)$}

All individuals begin their labor market careers in the state of unemployment (the labor market participation decision is not explicitly considered). Therefore, the ex ante value of the labor market career is the value of unemployed search, or $V_{n}(m)$. This measure of welfare is well-motivated if the conceptual experiment is to select a fixed minimum wage at the time that a reference cohort enters the labor market. For purposes of theoretical and empirical analysis it also has the advantage of being a scalar measure. We also note that since the unemployed have the lowest value of labor market participation, $V_{n}(m)$ can be considered a Rawlsian welfare measure.

We continue our example by considering the impact of minimum wages on some of the welfare measures discussed in this Section. Figures 4.a and 4.b contain plots of the value of search for each value of $m$ in the interval $[0,10]$ for the two cases of $\alpha=.3$ and $\alpha=.6$. For the case of $\alpha=.3$, to maximize the value of search a very high value of $m$ (8.02) is indicated. Conversely, for the relatively high bargaining power value of $\alpha=.6$ all binding minimum wages reduce the value of search. Therefore the optimal value of $m$ in this case is anything less than $\rho V_{n}(0)$.

\subsubsection{Ex Post Welfare $(W C 2)$}

Another conceptual welfare experiment, in some sense more "realistic," examines the effects of the imposition of a new minimum wage $m^{\prime}$ on the welfare of members of a cohort that has already entered the labor market and faces an environment $\Psi$ and a current minimum wage of $m$. We will assume throughout that the imposition of the new minimum wage is completely unanticipated by labor market participants.

Under any given minimum wage rate $m$ and labor market environment $\Psi$ we can assign a unique labor market value to each $\theta, \theta \in R_{+}$, and we will denote this value by $S(\theta ; m)$. For individuals with (hypothetical) draws of $\theta<m$, the value of the state is that of continued search. For those

with draws of $\theta$ greater than or equal to $m$ and less than $\hat{\theta}(m)$ the value of the state is that of employment at a wage of $m$, and for all values $\theta \geq \hat{\theta}(m)$, the value of the state is that associated with being employed at a wage $w\left(\theta, \rho V_{n}(m)\right)=\alpha \theta+(1-\alpha) \rho V_{n}(m)$. To summarize, we have:

$$
S(\theta ; m)=\left\{\begin{array}{clc}
V_{n}(m) & \Leftrightarrow & \theta<m \\
V_{e}(\theta ; m) & \Leftrightarrow & m \leq \theta<\hat{\theta}(m) . \\
V_{e}\left(w\left(\theta, \rho V_{n}(m)\right) ; m\right) & \Leftrightarrow & \hat{\theta}(m) \leq \theta
\end{array}\right.
$$

The formal definition of Pareto improvement is then given by: 
Definition 1 (WC2) Labor market participants in a market $\Psi$ with a minimum wage $m$ enjoy a welfare gain from the unanticipated imposition of a minimum wage $m^{\prime}$ if and only if $S\left(\theta ; m^{\prime}\right) \geq$ $S(\theta ; m)$ for all $\theta \in R_{+}$and $S\left(\theta ; m^{\prime}\right)>S(\theta ; m)$ for $\theta \in \varsigma \subseteq R_{+}$, where $\varsigma$ is of positive measure.

When there is no binding minimum wage, so that $m \leq \rho V_{n}(0)$, we can without loss of generality set $m=0$. In this case, any match draw $\theta<\rho V_{n}(0)$ results in a match not being formed, and therefore the value of such a draw is the value of (continued) search. When the match is at least as large as $\rho V_{n}(0)$, then the value of the draw is given by the value of being employed at the wage generated by $\theta$. Then

$$
S(\theta ; 0)=\left\{\begin{array}{cl}
V_{n}(0) & \Leftrightarrow \theta<\rho V_{n}(0) \\
V_{e}\left(w\left(\theta, \rho V_{n}(0)\right) ; 0\right) & \Leftrightarrow \theta \geq \rho V_{n}(0)
\end{array}\right.
$$

Definition $W C 2$ clearly refers to Pareto-improving minimum wage changes, but when interpreting this criterion within the context of our model it is important to bear in mind that it does not refer to individuals, but rather to labor market states. The condition requires that for any labor market state any agent could occupy at any moment in time, the change to the binding minimum wage $m^{\prime}$ will either improve her welfare at this moment in time or will at least leave her indifferent between facing the binding minimum wage $m^{\prime}$ or none at all.

While the criterion refers only to improvements in the current values of participating in the labor market, due to the assumption that the population is homogeneous (in the sense that all individuals face the same labor market environment $\Psi$ ) there is a strong dynamic consistency to this criterion as well. Since each individual will occupy all labor market states at some point in their (infinitely-long) labor market career, the condition states that the individual will also be at least as well off in every future labor market state they can occupy under the minimum wage $m^{\prime}$ as they would be in the absence of a binding minimum wage. It is perhaps surprising that such a seemingly strong condition can be satisfied for "reasonable" values of the labor market environment $\Psi$ and choices of $m^{\prime}$.

When there doesn't currently exist a binding minimum wage (e.g., $m=0$ ), we have derived the following necessary and sufficient condition for $m^{\prime}$ to be Pareto-improving (see Flinn (2003)).

Proposition 2 The imposition of a minimum wage $m^{\prime}$ in a labor market that does not have one is welfare-improving under WC2 if and only if

$$
V_{n}\left(m^{\prime}\right) \geq V_{n}(0)+\frac{\alpha\left(m-\rho V_{n}(0)\right)}{\rho+\eta} .
$$

When a binding minimum wage already exists the necessary and sufficient condition for a Pareto improvement is slightly more complicated. The following result is also taken from Flinn (2003).

Proposition 3 The imposition of a new minimum wage $m^{\prime}$ in a labor market that currently has a binding minimum wage $m$ is welfare-improving under WC2 if and only if

$$
V_{n}\left(m^{\prime}\right) \geq V_{n}(m)+\frac{\alpha\left(m^{\prime}-\rho V_{n}(m)\right)}{\rho+\eta}, \hat{\theta}(m) \leq m^{\prime}
$$

or

$$
V_{n}\left(m^{\prime}\right) \geq \frac{m+\eta V_{n}(m)}{\rho+\eta}, m<m^{\prime}<\hat{\theta}(m)
$$


As can be seen from inspection of both of these cases, a necessary condition for $m^{\prime}$ to result in a Pareto improvement over $m$ is that the value of the unemployed state must be greater under $m^{\prime}$ than under $m$, or $V_{n}\left(m^{\prime}\right)>V_{n}(m)$. Thus improvement with respect to $W C 2$ implies improvement with respect to $W C 1$, but the converse is not true. In other words, if we find that a minimum wage change was not welfare-improving using $W C 1$, then it could not be the case that $m^{\prime}$ Pareto-dominates $m$. If we find that $m^{\prime}$ is better than $m$ using $W C 1$, we cannot claim that $m^{\prime}$ Pareto-dominates $m$.

It is not possible to find a "Pareto optimal" minimum wage, i.e., one for which the value of each possible match value $\theta$ is no less under that minimum wage than under any other. Therefore, it only makes sense to use the criterion $W C 2$ when comparing two minimum wage rates. In Figures 4.c and 4.d we plot the state values $S(\theta ; m)$ for $m=0$ and $m=7$ for both of the values of $\alpha$ we are considering. When workers have little bargaining power (Figure 4.c), the binding minimum wage of 7 improves the value of any possible draw of $\theta$, even for those that imply the "loss" of a job. The situation is very different for those with relatively high bargaining power. In this case, only match values in a small range beginning at 7 show an improvement under the binding minimum wage. It is not difficult to construct examples in which all $\theta$ have lower state values under a higher minimum wage than under a lower one.

\subsubsection{Average Welfare $(W C 3)$}

It is often the case that average welfare measures are employed in applications, where the averaging can be of a relatively general form. A characterization of this type of welfare comparison is as follows.

Definition 4 (WC3) A minimum wage $m^{\prime}$ is at least as good as a minimum wage $m$ if and only if

$$
\int S\left(\theta ; m^{\prime}\right) d \mu\left(\theta \mid m^{\prime}\right) \geq \int S(\theta ; m) d \mu(\theta \mid m),
$$

for some given weighting functions $\mu\left(\theta \mid m^{\prime}\right)$ and $\mu(\theta \mid m)$, where

$$
\begin{aligned}
\mu(\theta \mid x) & \geq 0, \forall \theta \geq 0 \\
\int d \mu(\theta \mid x) & =1
\end{aligned}
$$

for $x=m, m^{\prime}$.

We utilize the steady state distribution of types in the population under policy $x$ as the weighting function $\mu(\theta \mid x)$. Under binding minimum wage $m$, we know that the probability that an individual will be in the unemployment state at any arbitrary point in time is given by $\eta /(\eta+\lambda \tilde{G}(m))$. We can arbitrary assign any unemployed individual a value of $\theta$ less than $m$ in forming the valuation of their labor market state, so without loss of generality assign the unemployment state the $\theta$ value of $m / 2$. Then the "weight" attached to the state $\theta=m / 2$ is given by

$$
\mu\left(\frac{m}{2} \mid m\right)=\frac{\eta}{(\eta+\lambda \tilde{G}(m))} .
$$

For individuals who are employed at wage $m$, we know that their match value is in the interval $[m, \hat{\theta}(m))$. Any value of $\theta$ in the interval $[m, \hat{\theta}(m))$ can be arbitrarily assigned to such an individual, 
so without loss of generality let us assign the value $\theta=(m+\hat{\theta}(m)) / 2$ for this event. Then we have

$$
\begin{aligned}
\mu\left(\frac{m+\hat{\theta}(m)}{2} \mid m\right)=p(\theta \in[m, \hat{\theta}(m)) \mid e) \times p(e) & =\frac{\tilde{G}(m)-\tilde{G}(\hat{\theta}(m))}{\tilde{G}(m)} \times \frac{\lambda \tilde{G}(m)}{\eta+\lambda \tilde{G}(m)} \\
& =\frac{\lambda(\tilde{G}(m)-\tilde{G}(\hat{\theta}(m))}{\eta+\lambda \tilde{G}(m)} .
\end{aligned}
$$

Finally, the likelihood of observing an individual with a value of $\theta \geq \hat{\theta}(m)$ in the steady state is given by

$$
\begin{aligned}
f(\theta \mid \theta \geq \hat{\theta}(m), e) \times p(\theta \geq \hat{\theta}(m) \mid e) \times p(e) & =\frac{g(\theta)}{\tilde{G}(\hat{\theta}(m))} \times \frac{\tilde{G}(\hat{\theta}(m))}{\tilde{G}(m)} \times \frac{\lambda \tilde{G}(m)}{\eta+\lambda \tilde{G}(m)} \\
& =\frac{\lambda g(\theta)}{\eta+\lambda \tilde{G}(m)}, \theta \geq \hat{\theta}(m),
\end{aligned}
$$

so in this case we have

$$
\mu(\theta \mid m)=\frac{\lambda g(\theta)}{\eta+\lambda \tilde{G}(m)}, \theta \geq \hat{\theta}(m)
$$

Using this definition of the weighting function, and assigning all $\theta$ values not explicitly referred to above a weight of 0 , the welfare value of a minimum wage under criterion $W C 3$ is given by

$$
\begin{aligned}
\int S(\theta ; m) d \mu(\theta \mid m)= & V_{n}(m) \times \frac{\eta}{\eta+\lambda \tilde{G}(m)} \\
& +V_{e}\left(\frac{m+\hat{\theta}(m)}{2} ; m\right) \times \frac{\eta+\lambda(\tilde{G}(m)-\tilde{G}(\hat{\theta}(m))}{\eta+\lambda \tilde{G}(m)} \\
& +\int_{\hat{\theta}(m)} V_{e}\left(w\left(\theta, \rho V_{n}(m)\right) ; m\right) \frac{\lambda g(\theta)}{\eta+\lambda \tilde{G}(m)} d \theta .
\end{aligned}
$$

Under this criterion it is clear that the impact of the policy both on the state valuations and the steady state distribution determine the welfare value of the policy (these are simultaneously determined in any event). As a result, conditions under which $m^{\prime}$ will have an expected steady state welfare value greater than that associated with $m$ are difficult to derive and are not particularly illuminating. Of most interest for our argument is the fact that $V_{n}\left(m^{\prime}\right)>V_{n}(m)$ does not imply that $\int S\left(\theta ; m^{\prime}\right) d \mu\left(\theta \mid m^{\prime}\right)>\int S(\theta ; m) d \mu(\theta \mid m)$. While there is no direct theoretical link between $W C 1$ and $W C 3$, the policy experiments conducted in Section 6 will illustrate that similar values of the "optimal" minimum wage are selected using either of them.

In Figures 4.e and 4.f we illustrate the relationship between the expected steady state welfare level and the minimum wage rate for our two values of $\alpha$. While we not able to formally characterize the relationship between $V_{n}(m)$ and average steady state welfare, in our examples the welfare criteria behave in a similar manner with respect to changes in $m$. Due to the absence of formal results, we cannot speculate as to whether this similarity could be expected to hold in other labor market environments $\Psi$.

\subsection{Employment Effects}

What of standard employment effects in this model? Clearly, if members of a homogeneous group are labor market participants prior to the imposition of the minimum wage, then after the imposition 
of a binding minimum wage the employment rate will decrease. In particular, the change in the steady state probability of employment will be

$$
\frac{\lambda \tilde{G}(m ; \mu)}{\eta+\lambda \tilde{G}(m ; \mu)}-\frac{\lambda \tilde{G}\left(\rho V_{n} ; \mu\right)}{\eta+\lambda \tilde{G}\left(\rho V_{n} ; \mu\right)}<0
$$

since $m>\rho V_{n} \cdot{ }^{5}$ Thus in this case the model is not consistent with some recent findings of small positive employment effects of minimum wage increases, but is consistent with the large number of studies of minimum wage effects on employment using cross-sectional, panel, and time series data. One of the major points of our analysis is that the imposition of a minimum wage has the potential to be welfare-increasing, even though employment effects are always negative, at least for groups participating in the market both before and after the change.

\subsection{Effects on the Wage Distribution}

Within this model the effects of imposing a minimum wage on the accepted wage distribution are complex, though the Nash bargaining structure of the problem does impose a number of restrictions on the relationship between the pre- and post-change distributions. First, and most obvious, the minimum observed wage will always increase in response to the imposition of a binding minimum wage or when a binding minimum wage is increased.. Second, if the (new) minimum wage is welfare increasing in the sense of increasing the value of unemployed search, then the new observed wage distribution will stochastically dominate the original one. If the new minimum wage reduces the value of search, the accepted wage distribution will either first order stochastically dominate the old one or the respective c.d.f.s will cross once. We analyze these effects and others in detail in Flinn (2003); what follows in this subsection is a brief summary of some of the more important results obtained in that paper. The results refer to comparisons between wage offer distributions observed under two different minimum wages, $m$ and $m^{\prime}$, where $m<m^{\prime}$. In order to apply them to the situation in which the labor market moves from not having a binding minimum wage to imposing a binding one, simply set $m=0$. Proofs of all propositions can be found in Flinn (2003).

Proposition 5 Let the wage distribution under the minimum wage $m^{\prime}$ be given by $F_{2}(w)$ and that under $m$ be given by $F_{1}(w)$, for $m<m^{\prime}$. Then $F_{2}$ first order stochastically dominates $F_{1}$ iff

$$
\frac{\tilde{G}(m)}{\tilde{G}\left(m^{\prime}\right)} \geq \frac{\tilde{G}\left(\frac{z-(1-\alpha) \rho V_{n}(m)}{\alpha}\right)}{\tilde{G}\left(\frac{z-(1-\alpha) \rho V_{n}\left(m^{\prime}\right)}{\alpha}\right)} \text { for all } z \geq m^{\prime} .
$$

This result can be used to derive the following sufficient condition for the "new" wage distribution to first order stochastically dominate the original one.

Corollary 6 If $V_{n}\left(m^{\prime}\right)>V_{n}(m)$, then $F_{2}$ first order stochastically dominates $F_{1}$.

Unfortunately, however, this condition is only sufficient. If are goal is to infer the direction of the welfare change (i.e., $\operatorname{sgn}\left(V_{n}\left(m^{\prime}\right)-V_{n}(m)\right)$ from the relationship between the two observed wage distributions, the most useful result available to us is the following.

Corollary 7 If $F_{2}$ does not first order stochastically dominate $F_{1}$ then $\tilde{V}_{n}\left(m^{\prime}\right)<\tilde{V}_{n}(m)$.

\footnotetext{
${ }^{5}$ Clearly a similar negative effect will be observed when moving from a binding $m$ to a larger value $m^{\prime}$.
} 
In reality, $F_{2}$ may not first order stochastically dominate $F_{1}$ due to a variety of features of the two distribution functions. Our model specification places restrictions on the way in which FOSD can fail. In particular, if $F_{2}$ does not FOSD $F_{1}$, there must exist some $x^{*}$ such that $F_{2}(x) \leq F_{1}(x)$ for all $x \leq x^{*}$ and $F_{2}(x)>F_{1}(x)$ for all $x>x^{*}$. That is, the c.d.f.s should intersect either never (in which case $F_{2}$ first order stochastically dominates $F_{1}$ ) or once and only once (in the case of failure of FOSD). ${ }^{6}$ Multiple crossings of the c.d.f.s could be produced only by sampling variability or model misspecification.

Our model also allows us to rigorously define what is commonly termed "spillover" in the minimum wage literature. ${ }^{7}$ Consider a wage rate $w$ such that $w>m^{\prime}>m$. Then under either value of the minimum wage the density of accepted wages at $w$ exists. ${ }^{8}$ Consider the ratio of the density at $w$ under $m^{\prime}$ and $m$, which is in essence a likelihood ratio. Then we define

$$
\begin{aligned}
L\left(w ; m, m^{\prime}\right) & =\frac{\frac{\alpha^{-1} g\left(\tilde{\theta}\left(w, V_{n}\left(m^{\prime}\right)\right)\right)}{\tilde{G}\left(m^{\prime}\right)}}{\frac{\alpha^{-1} g\left(\tilde{\theta}\left(w, V_{n}(m)\right)\right)}{\tilde{G}(m)}} \\
& =\frac{\tilde{G}(m) \times g\left(\tilde{\theta}\left(w, V_{n}\left(m^{\prime}\right)\right)\right)}{\tilde{G}\left(m^{\prime}\right) \times g\left(\tilde{\theta}\left(w, V_{n}(m)\right)\right)} .
\end{aligned}
$$

The ratio $\tilde{G}(m) / \tilde{G}\left(m^{\prime}\right)$ in $L\left(w ; m, m^{\prime}\right)$ we might refer to as the truncation effect of the minimum wage change. Since $\tilde{G}(m)>\tilde{G}\left(m^{\prime}\right)$, this effect is always greater than 1 and is independent of the value of $w, w>m^{\prime}$; we will write it as $T\left(m, m^{\prime}\right)$. We view this effect on the ratio of wage densities at $w$ as rather mechanical and uninteresting. Instead, what we will refer to as the spillover effect is the term

$$
S\left(w ; m, m^{\prime}\right)=\frac{g\left(\tilde{\theta}\left(w, V_{n}\left(m^{\prime}\right)\right)\right)}{g\left(\tilde{\theta}\left(w, V_{n}(m)\right)\right)} .
$$

In this way we have constructed a decomposition of the likelihood ratio of the wage density at $w$ before and after the wage change, which is

$$
L\left(w ; m, m^{\prime}\right)=T\left(m, m^{\prime}\right) S\left(w ; m, m^{\prime}\right) .
$$

It will be convenient to work with an additive decomposition of the log likelihood ratio, or

$$
\ln L\left(w ; m, m^{\prime}\right)=\ln T\left(m, m^{\prime}\right)+\ln S\left(w ; m, m^{\prime}\right) .
$$

Using the logarithmic decomposition, it is clear that the truncation effect shifts $\ln L$ by the uniform amount $\ln T\left(m, m^{\prime}\right)$. Furthermore we know that $\ln T\left(m, m^{\prime}\right)>0$ for any two binding minimum wages $m^{\prime}>m$. Our main interest is in the manner in which the shape of the wage density above $m^{\prime}$ changes with a change in the minimum wage. We will assess this by looking at the manner in which $\ln L\left(w ; m, m^{\prime}\right)$ varies in $w$. That is, we are interested in

$$
\frac{\partial \ln L\left(w ; m, m^{\prime}\right)}{\partial w}=\frac{\partial \ln S\left(w ; m, m^{\prime}\right)}{\partial w} .
$$

We work with the logarithm of the likelihood ratio so that the truncation effect can be ignored.

\footnotetext{
${ }^{6}$ The single-crossing property is not sufficient to produce second order stochastic dominance.

${ }^{7}$ The definition of spillover that we utilize and analyze within the context of this specific model is the same as the one employed by Dinardo et al. (1996).

${ }^{8}$ For purposes of this discussion we assume that the matching distribution has unbounded support, which implies that the wage distribution will share this characteristic as well whenever $\alpha>0$.
} 
Definition 8 The quantity $\partial \ln S\left(w ; m, m^{\prime}\right) / \partial w$ is called the shape perturbation at $w$ associated with the minimum wage increase from $m$ to $m^{\prime}$. We denote this quantity by $S P\left(w ; m, m^{\prime}\right)$.

In general, minimum wage changes result in changes in the shape of the density above the new minimum wage. It is interesting to consider when this would not be the case. We begin with one readily checkable sufficient condition for the absence of shape perturbations.

Proposition 9 Assume that $G(\theta)$ is continuously differentiable on its support $Q \subseteq R_{+}$, where $Q$ is a connected set. Then there is no spillover when moving from minimum wage $m$ to $m^{\prime}$ if and only if at least one of the following:

1. $V_{n}\left(m^{\prime}\right)=V_{n}(m)$

2. $g(\theta)=\tau^{-1} \exp (\beta \theta)$ for all $\theta \in Q$, where $\tau=\tau(\beta, Q)=\int_{Q} \exp (\beta x) d x<\infty$.

The types of distributions which satisfy condition 2 are relatively familiar ones. When $\beta=0$, then we have $g(x)=\tau^{-1}$ on $Q$, which implies that $Q$ is a finite interval $[\underline{\theta}, \bar{\theta}]$, with $0 \leq \theta<\theta<\infty$, so that $\tau=[\bar{\theta}-\underline{\theta}]$. In this case $G$ corresponds to a uniform distribution. When $\beta<0$, we have the case of a negative exponential distribution. When $Q=R_{+}$, then $\tau=|\beta|^{-1}$. When $Q$ is a bounded subset of $R_{+}$, then $g$ is a truncated negative exponential density. Finally, when $\beta>0$, for integrability of the density we require that $Q$ be a bounded subset of $R_{+}$. In other analytic respects this case closely resembles that of $\beta<0$.

A result which follows immediately from condition 2 of the above proposition is the following

Proposition 10 If condition 2 of Proposition 9 is satisfied, then

$$
f(w ; m)=\delta\left(m, m^{\prime}\right) f\left(w ; m^{\prime}\right), \forall w>m^{\prime}>m,
$$

where

$$
\delta\left(m, m^{\prime}\right)=\frac{\tilde{G}\left(m^{\prime}\right)}{\tilde{G}(m)} \frac{g\left(-\frac{1-\alpha}{\alpha} \rho \tilde{V}_{n}(m)\right)}{g\left(-\frac{1-\alpha}{\alpha} \rho \tilde{V}_{n}\left(m^{\prime}\right)\right)} .
$$

In Flinn (2003) these results and others are used to characterize conditions under which changes in certain properties of observed wage distributions can be used to infer the direction of the welfare effects resulting from minimum wage changes. In that paper these results were used in an attempt to determine the welfare effects of the 1996 and 1997 minimum wage changes using nonparametric methods. In contrast, the parametric approach we adopt in this paper can be used to directly estimate these effects, albeit at the cost of making particular parametric assumptions. We provide these estimates below in Section 5 .

\subsection{Firm Welfare and Other Demand Side Effects}

To this point we have said little about the behavior and welfare of firms. The demand side of the model has been kept deliberately simple because the vast majority of the information available to us concerns the behavior and outcomes for individuals on the supply side of the market. As we will see below, however, a modicum of information from the demand side is required if we are to develop relatively robust methods for identifying key model parameters. In this subsection we provide a 
discussion of the impact of minimum wages on firm welfare and some summary statistics (for the economy) that will be used in some of the estimation strategies developed below.

Due to search frictions, matching heterogeneity, and the costless creation of job vacancies, firms earn positive profits in the short- and long-run. The model is silent as to the determination of the number of firms in the market, as it is as to the number of individuals participating on the supply side. Let the number of firms in the market be denoted $K$, and let the number of individuals participating be given by $N$; both $K$ and $N$ are assumed to be large enough that laws of large numbers are applicable. In the steady state the number of agents employed is

$$
N_{e}(m)=\frac{\lambda \tilde{G}(m)}{\eta+\lambda \tilde{G}(m)} N,
$$

and the average number of workers per firm is given by $A E(m)=N_{e}(m) / K$. The average instantaneous profit per worker at all firms under minimum wage $m$ is

$$
A P(m)=\int_{m}\left(\theta-w\left(\theta ; m, \rho V_{n}(m)\right)\right) d G(\theta) / \tilde{G}(m) .
$$

Then the steady state instantaneous profit of any firm is proportional to $\Pi(m)=A E(m) A P(m)$. Given the stationarity of the environment it seems natural to equate the value $\Pi(m)$ with the welfare of the representative firm.

Assuming existence of the matching density everywhere on the real line and the differentiability of the value of search with respect to $m$, we have

$$
\frac{\partial \Pi(m)}{\partial m}=\frac{\partial A E(m)}{\partial m} A P(m)+N_{e}(m) \frac{\partial A P(m)}{\partial m} .
$$

In terms of the employment effect, from our discussion above we know that it is unambiguously negative, with

$$
\begin{aligned}
\frac{\partial A E(m)}{\partial m} & =-\frac{N}{K} \frac{\lambda g(m)}{(\eta+\lambda \tilde{G}(m))^{2}} \\
& <0 .
\end{aligned}
$$

The impact on average profit per employee is given by

$$
\frac{\partial A P(m)}{\partial m}=\frac{g(m)}{\tilde{G}(m)} A P(m)-p(w=m)-(1-\alpha) \rho \frac{\partial V_{n}(m)}{\partial m} p(w>m),
$$

where $p(w=m)$ is the probability that an employed agent is paid the minimum wage and $p(w>m)$ is the probability that an employed worker is paid more than $m$. Note that under the model the first term on the right hand side of [13] must be positive; this reflects purely the selection effect of improving match quality by imposing a higher "standard." The second term is always negative and reflects the fact that all those receiving the minimum wage must now be paid more. The third term is of ambiguous sign. When the minimum wage change increases the value of search, and hence the welfare of individuals under welfare criterion $W C 1$, then this term must be negative. Since a minimum wage change may decrease the value of search, the firm may benefit from the minimum wage change among those workers paid more than the minimum wage due to the reduction in the value of their threat point. While there is a clear interpretation of the various impacts of a 
minimum wage change on worker welfare, clearly there is no way to sign the change without access to the primitive parameters that describe the labor market.

Using the model it is possible to analyze the impact of minimum wages on many potentially observable aggregate labor market characteristics. One of these statistics will be heavily exploited in the econometric analysis below because of its availability. Under the model, the share of total revenues that are used by firm $j$ for employee compensation given a minimum wage of $m$ is given by

$$
\pi_{W}^{j}(m)=\frac{\sum_{i \in E^{j}} w\left(\theta_{i} ; m, \rho V_{n}(m)\right)}{\sum_{i \in E^{j}} \theta_{i}}
$$

where $E^{j}$ is the set of employees at firm $j$ at some arbitrary point in time (and it is assumed that there is at least one). Dividing both by the numerator and denominator by the number of employees at the firm we get

$$
\pi_{W}^{j}(m)=\frac{\bar{w}^{j}}{\bar{\theta}^{j}}
$$

If the number of employees at firm $j$ is large enough for the law of large numbers to apply, then

$$
\pi_{W}^{j}(m) \doteq \frac{m p(w=m)+\int_{\frac{m-(1-\alpha) \rho V_{n}(m)}{\alpha}}\left(\alpha \theta+(1-\alpha) \rho V_{n}(m)\right) d G(\theta) / \tilde{G}(m)}{\int_{m} \theta d G(\theta) / \tilde{G}(m)} .
$$

If there exists no particular firm $j$ with enough employees for the law of large numbers to be applicable, it must be the case that the average wage share of revenues across all firms in the market, $\sum_{j} \pi_{W}^{j}(m) / K$, assumes a value arbitrarily close to the right hand side of [14] for a sufficiently large population of firms (i.e., as $K \rightarrow \infty$ ).

This brief description of the impact of minimum wages on firm outcomes has hopefully served two purposes. The first is to stress the point that minimum wage increases are not necessarily an anathema to firms. The second is that readily available statistics on the performance of firms are directly linked to the parameters of the model.

\section{Econometric Issues}

The model as formulated relies heavily on stationarity assumptions, which enable us to use what are essentially cross-sectional data sets to estimate dynamic models. The CPS point-in-time sample only contains information on the length of time individuals currently unemployed have been actively searching for a job and the hourly wage rate and/or gross weekly earnings and usual hours worked per week for those reporting that they are currently employed. No information is available on the length of time an employed individual has worked for their current employer. A substantial amount of demographic information is available for each individual as well, information which is sparingly used in most of the empirical analysis that we conduct.

We will begin our analysis by laying out the information content of the CPS data for purposes of estimating the labor market model developed above. We shall see that a major stumbling block to the estimation of such a model is the identification of the bargaining power parameter $\alpha$. The existence of this problem is widely recognized among researchers who have tried to estimate bargaining models (see, e.g., Flinn and Heckman (1982), Kapteyn and Kooreman (1990), and 
Eckstein and Wolpin (1993)). ${ }^{9}$ This is a particularly vexing problem in this application since one of our goals is to conduct policy experiments, and the results of these experiments are likely to depend critically on the bargaining power parameter $\alpha$.

We will explore a number of ways in which the model can be estimated using the CPS data available to us (and in one case auxiliary information from other sources), although some of these will be mentioned only in passing. Possible approaches to the under-identification problem include: (1) normalization, (2) the use of demand-side information, (3) the use of observable heterogeneity in conjunction with exclusion restrictions, and (4) natural experiments. Each approach has its drawbacks, though we shall argue that method (2) provides the most "robust" method for model identification if one is willing to make some distributional assumptions. We shall present model estimates based approaches (1) and (2).

In most empirical implementations of behavioral search models an allowance is made for measurement error in the wage data (but not in the duration measures). Allowing for measurement error may be indicated when the sample contains very low wage observations, which without measurement error would imply a perhaps incredibly small reservation wage. When allowing for on-the-job search, which we do not do here, measurement error is required for the model of expected wealth maximization to be consistent with direct job-to-job transitions in which the destination job offers a lower wage than the job which was left.

In the presence of a binding minimum wage, it is not possible to add "classical" measurement error to the model. If the reported wage distribution is assumed to be given by the convolution of the distribution of actual wages and some continuously distributed i.i.d. random variable, then even though the true wage distribution is of the mixed continuous-discrete type, the observed wage distribution will be absolutely continuous. Since this is inconsistent with even the most casual inspection of the data (see Figures 1.a and 1.c, for example), classical measurement error cannot be introduced. While one could consider allowing for "contamination" in the data, i.e., that some unknown proportion $\tau$ of wages are measured with classical error while the remainder are not, the estimation of $\tau$ would seem not to be possible without further arbitrary assumptions. As a result, we assume throughout that all observations are measured exactly. While we have already noted that a large number of observations are "heaped" at focal points, our hope is that the regular pattern of the heaping process will not bias our estimates to any great degree.

The likelihood function based on the CPS data is constructed as follows. Each individual observation can be characterized by the pair of observations $\left(t_{i}, w_{i}\right), i=1, \ldots, N$. The variable $t_{i}$ is the length of the on-going spell of unemployed search, which is positive if the sample member is unemployed at the time of the survey and is otherwise equal to 0 . If the individual is employed, the hourly wage $w_{i}$ is recorded. If the individual is paid an hourly wage, $w_{i}$ corresponds to the hourly wage rate they report. If the individual is not paid on an hourly basis, the hourly rate is imputed by dividing the gross weekly wage by the usual hours of work per week. This imputation procedure is standard, but is particularly problematic in this application because of the likely undercount of individuals paid exactly the minimum wage that will result. On the positive side, the vast majority

\footnotetext{
${ }^{9}$ Recent notable attempts to estimate worker bargaining power are Cahuc et al (2002) and Yashiv (2003). Cahuc et all use matched employer-employee data from France with a different matching structure than that employed here to estimate this parameter. They are greatly helped by having limited information from both sides of the market. Yashiv estimates a bargaining power parameter using aggregate time series data for Israel. In the sense that these series represent equilibrium outcomes for the market as a whole, he also does not face the problem of having observations only from the supply side though he must confront the typical identification problem of distinguishing supply and demand behaviors.
} 
of our sample members report an hourly wage rate so that the imputation procedure has to be utilized for less than 20 percent of the sample in any given year.

There are essentially three components of the likelihood function, one for the unemployed, one for the employed paid the minimum wage, and one for those paid more than the minimum. Let us first consider the contribution of a sample member who is unemployed with an on-going unemployment spell of length $t$. Given being in the unemployed state, the likelihood of finding someone in an on-going spell of length $t$ in the steady state is given by $r_{u}(t)=\tilde{F}_{u}(t) / E(t)$, where $\tilde{F}_{u}$ is the population survivor function of unemployment durations and $E(t)$ is the population mean duration of unemployment spells. When the population unemployment spell duration distribution is of the negative exponential form, it is well-known that $r_{u}(t)$ is equal to the population density. Thus, given unemployment, the density associated with an on-going spell length of $t$ is

$$
f_{u}(t \mid u)=\lambda \tilde{G}(m) \exp (-\lambda \tilde{G}(m) t)
$$

where the acceptance match value of $m$ presumes the existence of a binding minimum wage (in the absence of one $m$ is replaced by $\rho V_{n}$ in the above expression). Using standard ergodic results, the steady state probability of unemployment is given by

$$
p(u)=\frac{\eta}{\eta+\lambda \tilde{G}(m)}
$$

so that the joint likelihood of $(t, u)$ is given by

$$
f(t, u)=\frac{\eta \lambda \tilde{G}(m) \exp (-\lambda \tilde{G}(m) t)}{\eta+\lambda \tilde{G}(m)} .
$$

Next we consider the likelihood contribution of an individual who is paid the minimum wage $m$. We will assume that whenever two or more population members are paid the minimum wage $m$ that the minimum wage is binding for their population type. Assuming that it is, conditional on employment the likelihood of being paid $m$ is given by

$$
p(w=m \mid e)=\frac{\tilde{G}(m)-\tilde{G}\left(\frac{m-(1-\alpha) \rho V_{n}(m)}{\alpha}\right)}{\tilde{G}(m)} .
$$

Therefore the likelihood of observing $(w=m, e)$ is

$$
p(w=m, e)=\frac{\lambda\left[\tilde{G}(m)-\tilde{G}\left(\frac{m-(1-\alpha) \rho V_{n}(m)}{\alpha}\right)\right]}{\eta+\lambda \tilde{G}(m)} .
$$

The contribution of individuals paid more than the minimum wage is determined as follows. To be paid a wage $w>m$, it must be the case that the match value exceeds $\left(m-(1-\alpha) \rho V_{n}(m)\right) / \alpha$. Thus the likelihood of being a paid a wage $w$ given employment and $w>m$ is

$$
f(w \mid w>m, e)=\frac{\frac{1}{\alpha} g\left(\frac{w-(1-\alpha) \rho V_{n}(m)}{\alpha}\right)}{\tilde{G}\left(\frac{m-(1-\alpha) \rho V_{n}(m)}{\alpha}\right)} .
$$

Furthermore, the probability that a sample member is paid a wage greater than $m$ given that she is employed is

$$
p(w>m \mid e)=\frac{\tilde{G}\left(\frac{m-(1-\alpha) \rho V_{n}(m)}{\alpha}\right)}{\tilde{G}(m)},
$$


so that the likelihood contribution for such an individual is given by

$$
\begin{aligned}
f(w, w>m, e) & =\frac{\frac{1}{\alpha} g\left(\frac{w-(1-\alpha) \rho V_{n}(m)}{\alpha}\right)}{\tilde{G}\left(\frac{m-(1-\alpha) \rho V_{n}(m)}{\alpha}\right)} \frac{\tilde{G}\left(\frac{m-(1-\alpha) \rho V_{n}(m)}{\alpha}\right)}{\tilde{G}(m)} \frac{\lambda \tilde{G}(m)}{\eta+\lambda \tilde{G}(m)} \\
& =\frac{\frac{\lambda}{\alpha} g\left(\frac{w-(1-\alpha) \rho V_{n}(m)}{\alpha}\right)}{\eta+\lambda \tilde{G}(m)} .
\end{aligned}
$$

The log likelihood for the parameters describing the labor market environment of a given population can be written as

$$
\begin{aligned}
\ln L & =N[\ln (\lambda)-\ln (\eta+\lambda \tilde{G}(m))]+N_{u}[\ln (\eta)+\tilde{G}(m)] \\
& -\lambda \tilde{G}(m) \sum_{i \in U} t_{i}+N_{M} \ln \left(\tilde{G}(m)-\tilde{G}\left(\frac{m-(1-\alpha) \theta^{*}}{\alpha}\right)\right) \\
& -N_{H} \ln (\alpha)+\sum_{i \in H} \ln \left(g\left(\frac{w_{i}-(1-\alpha) \theta^{*}}{\alpha}\right)\right)
\end{aligned}
$$

where $N$ is the number of individuals in the sample, $N_{U}$ is the number of unemployed, $N_{M}$ is the number paid the minimum wage, $N_{H}$ is the number paid more than the minimum wage, $U$ is the set of indices of sample members who are unemployed, $H$ is the set of indices of sample members who are paid more than the minimum wage, and $\theta^{*} \equiv \rho V_{n}(m)$ is the "implicit" reservation wage in this population. Recall that $G$ will be assumed to belong to a parametric family, so that it can be characterized in terms of a finite-dimensional parameter vector $\tau$. Then the parameters that appear "directly" in the $\log$ likelihood are $\lambda, \eta, \tau, \alpha$, and $\theta^{*}$. The value $\theta^{*}$ is not a primitive parameter per se, but rather is a scalar that is determined endogenously and is a function of all the parameters of the model, including $\rho$ and $b$. For purposes of estimation, however, we shall treat it as a parameter of the model. ${ }^{10}$

\subsection{Model Identification}

We now present a discussion of the identification of the model using only CPS data. We begin by considering the case in which there is no binding minimum wage. After describing the identification problems faced in this case we will be in a better position to understand the situation in which a binding minimum wage is present.

Before we begin, let us review the identification results of Flinn and Heckman (1982), which are particularly relevant here. They demonstrated that the basic search model with only accepted wage and duration information is fundamentally underidentified, even in the absence of bargaining (i.e., $\alpha=1$ ). In particular, they showed that a parametric assumption on $G$ was required, and that only certain distributions with support on a subset of $R_{+}$were identified even under parametric assumptions. In the case we consider here, that of parametric $G$ with support given by $R_{+}$, all of

\footnotetext{
${ }^{10}$ Treating $\theta^{*}$ is a parameter in the estimation does not imply that all of the behavioral restrictions are not being imposed. The model is inherently underidentified in the sense that $\theta^{*}$ is determined by $\rho$ and $b$ in addition to the other parameters that appear "directly" in [15]. Once we estimate $\theta^{*}$, we can assume a value for $\rho$ or $b$ and then (uniquely) solve for the other. Conversely, we can directly estimate $\rho$ or $b$ after fixing the value of the other, and our estimate of the parameter along with the others in the likelihood function will uniquely determine $\theta^{*}$. This last procedure is less attractive from a computational standpoint due to the necessity of imposing a complicated, implicit restriction on the parameter space so as to ensure that the (implied) value of $\theta^{*}$ be strictly less than $m$.
} 
this class of distributions satisfy what they term the "recoverability condition." Thus we need not consider this particular problem further.

They also demonstrated that the parameters $(\rho, b)$ were not individually identified, essentially since they both enter the likelihood function only through the critical value $\theta^{*}$. Because of this we can treat $\theta^{*}$ as a parameter to be estimated without loss of generality. When all parameter estimates have been obtained, the functional equation that determines $\theta^{*}$ may be used with point estimates of all identified parameters to "back out" the locus of estimates of $(\rho, b)$. This is the procedure followed here. Thus we do not discuss identification of $\rho$ and $b$ explicitly below, but instead consider identification of $\theta^{*}$ along with the other true primitive parameters of the model.

\subsubsection{No Minimum Wage}

We begin by writing the log likelihood function for this problem in a transparent manner:

$$
\ln L=N \ln h_{U}-N \ln D+N_{U} \ln \eta-h_{U} \sum_{i \in U} t_{i}+\sum_{i \in E} \ln \frac{\frac{1}{\alpha} g\left(\frac{w_{i}-(1-\alpha) \theta^{*}}{\alpha}\right)}{\tilde{G}\left(\theta^{*}\right)},
$$

where $h_{U} \equiv \lambda \tilde{G}\left(\theta^{*}\right)$ and $D \equiv \eta+h_{U}$. Just as $\eta$ is the hazard rate from the employment state, $h_{U}$ is the hazard rate from the unemployment state; it is defined as the product of the rate of arrival of offers and the probability that an offer will be acceptable. Without auxiliary information to enable the identification of $G$, it will be impossible to disentangle the two components of $h_{U}$.

In discussing identification rigorously we will need to work with a class of matching distributions. For reasons that will become immediately clear, we will work with distributions with support $R_{+}$ that belong to a location-scale family. ${ }^{11}$ To be precise, the distribution $G(x ; c, d)$ belongs to a location-scale family if and only if

$$
G(x ; c, d)=G_{0}\left(\frac{x-c}{d}\right)
$$

where $c \in R$ and $d \in R_{+}$are two constants and $G_{0}$ is a known function. Assuming that $G$ is continuously differentiable on its support $R_{+}$, we have

$$
g(x ; c, d)=\frac{1}{d} g_{0}\left(\frac{x-c}{d}\right)
$$

as the probability density function of $x$. Many commonly used parametric distributions in economics belong to this class.

Given that $G$ belongs to a location-scale family, we can rewrite the last term in [16] as follows. Note that

$$
\frac{\frac{1}{\alpha} g\left(\frac{w_{i}-(1-\alpha) \theta^{*}}{\alpha} ; c, d\right)}{\tilde{G}\left(\theta^{*} ; c, d\right)}=\frac{\frac{1}{a d} g_{0}\left(\frac{w_{i}-(1-\alpha) \theta^{*}-\alpha c}{\alpha d}\right)}{\tilde{G}_{0}\left(\frac{\theta^{*}-c}{d}\right)} .
$$

Since $w^{*}=\theta^{*}$ when there is no binding minimum wage, we can write

$$
w^{*}=\alpha \theta^{*}-(1-\alpha) \theta^{*}
$$

\footnotetext{
${ }^{11}$ We could consider slightly more general classes of distributions, such as those that also possess a shape parameter, though the negative results of this section regarding the identifiability of $\alpha$ would only be reinforced.
} 
or

$$
\theta^{*}=\frac{w^{*}-(1-\alpha) \theta^{*}}{\alpha} .
$$

Substituting this expression into the denominator, we arrive at

$$
\frac{\frac{1}{a d} g_{0}\left(\frac{w_{i}-(1-\alpha) \theta^{*}-\alpha c}{\alpha d}\right)}{\tilde{G}_{0}\left(\frac{\frac{w^{*}-(1-\alpha) \theta^{*}}{\alpha}-c}{d}\right)}=\frac{\frac{1}{a d} g_{0}\left(\frac{w_{i}-(1-\alpha) \theta^{*}-\alpha c}{\alpha d}\right)}{\tilde{G}_{0}\left(\frac{w^{*}-(1-\alpha) \theta^{*}-\alpha c}{\alpha d}\right)} .
$$

This is the p.d.f. of a random variable generated by a truncated location-scale distribution, with lower truncation point $w^{*}$. The "new" location parameter is

$$
c^{\prime}=(1-\alpha) \theta^{*}+\alpha c
$$

and the new scale parameter is

$$
d^{\prime}=\alpha d .
$$

Since a super-consistent estimator for $\theta^{*}\left(=w^{*}\right)$ is available, the two parameters $c^{\prime}$ and $d^{\prime}$ will be a function of three unknown parameters: $\alpha, c$, and $d$.

Let us begin with the easiest problem, that of estimating the parameters $\eta$ and $h_{U}$. Taking the partial derivatives of $\ln L$ with respect to these two parameters, we obtain the following first order conditions

$$
\begin{aligned}
0 & =-\frac{N}{D}+\frac{N_{U}}{\eta} \\
0 & =\frac{N}{h_{U}}-\frac{N}{D}-\sum_{i \in U} t_{i}
\end{aligned}
$$

From the first equation we get

$$
\frac{\widehat{\eta}}{D}=\frac{N_{U}}{N},
$$

while manipulation of the second equation yields

$$
\begin{aligned}
\frac{N\left(D-\hat{h}_{U}\right)}{\hat{h}_{U} D} & =\sum_{i \in U} t_{i} \\
\Rightarrow \frac{N \eta}{\hat{h}_{U} D} & =\sum_{i \in U} t_{i} .
\end{aligned}
$$

After substituting [17] for $\frac{\eta}{D}$ in the above expression, we get

$$
\hat{h}_{U}=N_{U} / \sum_{i \in U} t_{i}
$$

Since $D=\eta+h_{U}$, we can now recover $\eta$ directly as

$$
\hat{\eta}=\frac{N_{U}^{2} / \sum_{i \in U} t_{i}}{N_{E}}
$$

Note that the estimators of $\eta$ and $h_{U}$ are independent of the specification of $G$. Of course, the specification of $G$ will be critical in "decomposing" $h_{U}$ into the products of the terms $\lambda$ and $\tilde{G}\left(\theta^{*}\right)$. 
We now consider the more difficult problem, that of identifying the parameters $\left(c, d, \alpha, \theta^{*}\right)$. A (super) consistent estimator for $\theta^{*}$ is trivial to obtain, as originally noted by Flinn and Heckman (1982), and is given by

$$
\hat{\theta}^{*}=\hat{w}^{*}=\min _{i \in E}\left\{w_{i}\right\} .
$$

This estimator converges at rate $N$ and therefore can be treated as a constant when it appears as an argument in any other estimator whose convergence rate is less than $N$. Since all other estimators in our model converge at rate $\sqrt{N}$, we can simply treat $\hat{\theta}^{*}$ as equal to $\theta^{*}$ when deriving the $\sqrt{N}$ asymptotic sampling distributions for these estimators.

Now we are left with the determination of the three unknown parameters that appear only in the wage distribution associated with the employed sample. For any given specification of $G_{0}$, and in fact even when $G_{0}$ is treated as unknown, there exist a number of consistent estimators of the location and scale parameters $c^{\prime}$ and $d^{\prime}$. Thus we assume with no loss of generality that consistent estimators of these parameters exist, and denote them by $\widehat{c^{\prime}}$ and $\widehat{d^{\prime}}$. With no prior information concerning $c, d$, or $\alpha$, no further progress toward identification of the individual parameters can be made. This is the fundamental underidentification problem associated with the asymmetric Nash bargaining model.

Proposition 11 Let $G$ belong to a location-scale family with parameters (c,d). Then a necessary condition for identification of $(c, d, \alpha)$ is that one of the parameters be expressible as a known, invertible function of the other two.

Proof: If there exists no restrictions relating $(c, d, \alpha)$, then consistent estimators of $c^{\prime}$ and $d^{\prime}$ are insufficient to determine all three parameters. Suppose that there exists one exact restriction on the parameter vector $(c, d, \alpha)$ of the form $0=r(c, d, \alpha)$, and without loss of generality let us use this restriction to determine a value of $\alpha$ given $(c, d)$, or $\alpha=\alpha(c, d)$. Then with consistent estimators for $c^{\prime}$ and $d^{\prime}$, we can solve for

$$
\begin{aligned}
& \widehat{c^{\prime}}=(1-\alpha(c, d)) \hat{\theta}^{*}+\alpha(c, d) c \\
& \widehat{d}^{\prime}=\alpha(c, d) d
\end{aligned}
$$

a system of two equations in two unknowns. Depending on the form of the function $\alpha(c, d)$ and the values of $\widehat{c^{\prime}}$ and $\widehat{d}^{\prime}$ there may exist no solution, a unique solution, or multiple solutions. We will say that the model is just identified if

$$
\begin{aligned}
c^{\prime} & =(1-\alpha(c, d)) \theta^{*}+\alpha(c, d) c \\
d^{\prime} & =\alpha(c, d) d
\end{aligned}
$$

has exactly one solution for all values of $(c, d)$ in the parameter space. Note that in this case we have replaced all estimated quantities with their limiting values, so that this should be interpreted as a large sample identification condition.

The most common restriction will be a direct one on one of the parameters $c$ or $d$. For example, if $c=0$ we have

$$
\begin{aligned}
& \widehat{c^{\prime}}=(1-\alpha) \hat{\theta}^{*} \\
& \widehat{d^{\prime}}=\alpha d .
\end{aligned}
$$


This is a two equation system in two unknowns, and yields estimators for $\alpha$ and $d$ of

$$
\begin{aligned}
& \hat{\alpha}=1-\frac{\widehat{c^{\prime}}}{\hat{\theta}^{*}} \\
& \hat{d}=\widehat{d}^{\prime} / \hat{\alpha} .
\end{aligned}
$$

While in small samples it is possible for these estimators to yield values outside of the parameter space, the likelihood of such an event will be a decreasing function of sample size.

We now establish why a restriction relating $(c, d, \alpha)$ is not sufficient to ensure identification. Assume that $G$ is negative exponential with support $R_{+}$. In this case the location parameter is 0 and the scale parameter $d>0$ completely characterizes the distribution. The likelihood of wage observation $w_{i}$ is given by

$$
\begin{aligned}
\frac{\frac{1}{a d} g_{0}\left(\frac{w_{i}-(1-\alpha) \theta^{*}}{\alpha d}\right)}{\tilde{G}_{0}\left(\frac{w^{*}-(1-\alpha) \theta^{*}}{\alpha d}\right)} & =\frac{\frac{1}{\alpha d} \exp \left(-\frac{w_{i}-(1-\alpha) \theta^{*}}{\alpha d}\right)}{\exp \left(-\frac{w^{*}-(1-\alpha) \theta^{*}}{\alpha d}\right)} \\
& =\frac{1}{\alpha d} \exp \left(-\frac{w_{i}-w^{*}}{\alpha d}\right) .
\end{aligned}
$$

Thus the wage distribution can only identify $\alpha d$, not the two components individually. This is the only continuous distribution with support $R_{+}$that I have found that is not identified under a restriction on $c$ or $d$ so that it may be a pathological case.

The fact that a restriction on $c$ or $d$ may be sufficient to enable identification of the other parameter characterizing $G$ and the bargaining power parameter $\alpha$ must not be viewed with too much optimism. While the knowledge that $c=0$, probably the most common type of restriction that will be imposed in this case, does lead to "theoretical" identification in large samples (as long as $G$ is not negative exponential), the small sample properties of the estimator tend to be poor. This last conclusion comes from conducting Monte Carlo experiments with a variety of scale distributions. If one fixes the scale parameter $(d)$, instead, the performance of an estimator for $\alpha$ tends to be better in reasonably-sized samples. Unfortunately, it is rarely the case that a researcher will feel comfortable fixing the value of a scale parameter a priori. Thus this case is not particularly germane for empirical researchers, and we are left with the impression that under most "reasonable" a priori restrictions on the parameters $(c, d)$, identification of the parameter $\alpha$ will remain tentative at best.

The fundamental reason for the identification problem is not hard to understand intuitively. From payoff data from one side of the market we are attempting to simultaneously determine the total size of the "pie" and the share received by the worker. While incredibly restrictive assumptions on the form of the underlying matching distribution may make $\alpha$ identifiable in theory, these restrictions are probably not believable and even imposing them is likely to produce an estimator with a large (though not infinite) standard error. We next consider whether having CPS information from a labor market with a binding minimum wage alleviates the identification problem to any extent. 


\subsubsection{Binding Minimum Wage}

In this case we can write the log likelihood function most transparently as

$$
\begin{aligned}
\ln L= & N \ln h_{U}-N \ln D+N_{U} \ln \eta-h_{U} \sum_{i \in U} t_{i} \\
& +N_{M} \ln \left(1-\frac{\tilde{G}\left(\frac{m-(1-\alpha) \theta^{*}}{\alpha}\right)}{\tilde{G}(m)}\right)+\sum_{i \in H} \ln \frac{\frac{1}{\alpha} g\left(\frac{w_{i}-(1-\alpha) \theta^{*}}{\alpha}\right)}{\tilde{G}(m)},
\end{aligned}
$$

where $h_{U}=\lambda \tilde{G}(m)$. The critical acceptance match value is now known to be equal to $m$, however we have to estimate the "implicit" critical match value $\theta^{*}$ along with the other primitive parameters of the model. We shall consider the identification of the parameters $\left(\eta, h_{U}, c, d, \alpha, \theta^{*}\right)$.

With respect to the rate parameters $\eta$ and $h_{U}$ no new issues arise. The maximum likelihood estimators for these two parameters are exactly as were given above for the case of no binding $m$.

Does the mass point at $m$ provide enough additional information to enable identification of $\alpha$ ? The answer to this question is no in general, and this is primarily due to the necessity of estimating $\theta^{*}$ simultaneously with the other parameters, something we did not have to do when there was no binding minimum wage. Let us begin by rewriting the terms involving $G$ in their location-scale form. For those paid the minimum wage, the term involving $G$ is written as

$$
1-\frac{\tilde{G}\left(\frac{m-(1-\alpha) \theta^{*}}{\alpha}\right)}{\tilde{G}(m)}=1-\frac{\tilde{G}_{0}\left(\frac{m-c^{\prime}}{d^{\prime}}\right)}{\tilde{G}_{0}\left(\frac{m-c}{d}\right)},
$$

where $c^{\prime}=(1-\alpha) \theta^{*}+\alpha c$ and $d^{\prime}=\alpha d$. For an individual $i$ who belongs to set $H$, their $\log$ likelihood contribution involving $G$ is written as

$$
\ln \frac{\frac{1}{d^{\prime}} g_{0}\left(\frac{w_{i}-c^{\prime}}{d^{\prime}}\right)}{\tilde{G}_{0}\left(\frac{m-c}{d}\right)}
$$

The fact that $(c, d)$ and $\left(c^{\prime}, d^{\prime}\right)$ appear independently in these expression may bring false optimism. If it were possible to consistently estimate these four terms, then we would be able to consistently estimate $\alpha$ and $\theta^{*}$ since

$$
\begin{aligned}
\alpha & =d^{\prime} / d \\
\theta^{*} & =\frac{c^{\prime}-\frac{d^{\prime}}{d} c}{1-\frac{d^{\prime}}{d}} .
\end{aligned}
$$

Thus all terms are clearly identified given consistent estimates of those four parameters. Unfortunately, it is not possible to consistently estimate $c$ and $d$ from these expressions. These terms only appear in the expression $\tilde{G}_{0}\left(\frac{m-c}{d}\right)$, which is the probability of encountering an acceptable match. Even given knowledge of this probability and the minimum wage $m$, there are in general a continuum of values of $(c, d)$ which can produce the same value of the argument $(m-c) / d$. Thus $(c, d)$ is not uniquely identified. Given this lack of uniqueness, we cannot uniquely determine $\alpha$ and $\theta^{*}$ from [19]. As was the case in the last subsection, identification may be obtainable if we are willing to impose a priori restrictions on the parameters $(c, d)$. For good reason, most analysts will be hesitant to resort to such devices. 
In the empirical results reported below, we have for the most part assumed that $G$ is lognormal. This is in keeping with much of the literature in empirical search or even earnings function estimation. We note that the lognormal (with support $R_{+}$) is a location-scale distribution in the natural logarithm space. That is,

$$
G(x ; c, d)=G_{0}\left(\frac{\ln (x)-c}{d}\right)
$$

where

$$
G_{0}(y)=\Phi(y)
$$

and $\Phi$ is the standard normal cumulative distribution function. Thus our identification discussion is germane to this case and we know that without further assumptions regarding $c$ and/or $d$ a consistent estimator of $\alpha$ cannot be found using only CPS data.

\subsection{Some Additional Identification Devices}

In empirical work utilizing a Nash-bargaining structure, it is common to assume symmetric bargaining, i.e., $\alpha=.5$ (e.g., Flinn and Heckman (1982), Eckstein and Wolpin (1993), and Del Boca and Flinn (1994)). Such a "normalization" is imposed both because the difficulty of identifying the parameter $\alpha$ has been apparent for some time, and also due to modeling considerations. In terms of the second point, recall that in our formulation of the behavioral model we assumed that both workers and firms shared a common effective discount rate. If we were to ignore the fact that the searcher has an "outside option" when bargaining with the firm, i.e., the value of continued search, bargaining over the match surplus could be placed in the framework of a Rubinstein (1982) alternating-offers game. It is well known that in the case of equal discount rates, the solution is equivalent to the symmetric Nash bargaining solution.

As the first step in the empirical analysis we simply restrict $\alpha=.5$ for every subpopulation considered. Besides conforming with common practice, this case serves as something of a benchmark. We were also interested in determining whether positive "optimal" minimum wages were ever indicated given this normalization. Roughly speaking, we know that the likelihood that binding minimum wages will improve the welfare of labor market participants is decreasing in $\alpha$. The empirical question we address is whether the restriction that $\alpha=.5$ effectively rules out a welfareimprovement role for minimum wages. Using the samples below, this indeed appears to be the case.

It is also possible to obtain identification of some parameters by redefining the parameter space with respect to observable characteristics of individuals. From the previous subsection, we know that if we sort sample members into a small number of observationally-distinguishable classes, identification of the labor market parameters for each class will not be possible. However, if we are willing to restrict the values of certain subsets of the primitive parameters to be constant across at least some of the classes, it will in general be possible to obtain identification of the reformulated model. The parameters that will be identified are those that are taken to be constant across at least some groups and those that are considered to be group-specific. Since our original model did not entail any across-group restrictions, this would have to be considered a nonequivalent reparameterization of the original model. It seems difficult to imagine that a researcher could specify a subset of primitive parameters that was common to a number of subpopulations in anything other

than an arbitrary manner. We have chosen not to estimate the model under these types of "exclusion restrictions" because we could not find any reasonable ones to impose. 
We have also attempted estimation of a subset of parameters by imposing an intertemporal restriction. A different nominal minimum wage was in place in March of 1996, 1997, and 1998. We allow for "approximately" neutral price changes across the years, and assume that changes in nominal minimum wages are unanticipated. Thus we view the wage distributions in each of these three years as being determined by a time invariant "real" match value distribution $G$, a time invariant bargaining power parameter $\alpha$, and a year-specific implicit reservation wage, $\theta_{t}^{*}$. At the imposition of a new minimum wage, all existing contracts are rebargained instantaneously, so that the current wage distribution reflects the influence of the contemporaneous $\theta_{t}^{*}$ and the existing "real" minimum wage $m_{t}$. We only use wage distribution information when imposing these restrictions, since even if agents continue to use stationary rules the contemporaneous probability of employment and durations of unemployed search will reflect past environmental conditions.

\subsection{Use of Demand Side Information}

Our analysis of minimum wages takes place in the context of a simple partial equilibrium model of the labor market. In the end it is not terribly surprising that identification of an equilibrium model using data from outcomes only for one side of the market is a daunting task. In this section we explore identification strategies that utilize both the CPS data and some information readily available from the financial statements of publicly held corporations.

We use some of the results derived above during our discussion of the welfare of firms to form an estimator of $\alpha$ jointly with $\gamma$, where $\gamma$ denotes all other model parameters. In particular, we recognize that the wage share of firm revenues, defined as $\pi_{W}^{j}(m)$, is a function of all of the primitive parameters of the model, including $\alpha$. The idea is to form a concentrated likelihood function in which an estimator for $\alpha$, written as a function of the parameter vector $\Gamma$ and an observed value of $\pi_{W}^{j}(m)$, is substituted into the "unconditional" likelihood function. From [14], we see that any $\alpha \in(0,1)$ and $\gamma \in \Gamma$ imply a unique value of the wage share of revenues, which we will now call simply $\pi$, thus

$$
\pi=\pi(\alpha ; \gamma, m)
$$

The function $\pi$ has the following features (for all $m$ ):

$$
\begin{aligned}
\lim _{\alpha \rightarrow 1} \pi(\alpha ; \gamma, m) & =1, \\
\lim _{\alpha \rightarrow 0} \pi(\alpha ; \gamma, m) & =\frac{m}{E(\theta \mid \theta \geq m)} \in(0,1],
\end{aligned}
$$

and

$$
\frac{\partial \pi(\alpha ; \gamma, m)}{\partial \alpha}=\frac{\int_{\frac{m-(1-\alpha) \rho V_{n}(m)}{\alpha}}\left(\theta-\rho V_{n}(m)\right) d G(\theta)}{\int_{m} \theta d G(\theta)} \in(0,1) .
$$

Then $\pi(\alpha ; \gamma, m):[0,1] \rightarrow\left[\frac{m}{E(\theta \mid \theta \geq m)}, 1\right]$. Furthermore, $\pi$ is monotone in $\alpha$. Let the observed value of $\pi$ be denoted by $\tilde{\pi}$. Then for $\tilde{\pi} \in\left(\frac{m}{E(\theta \mid \theta \geq m)}, 1\right)$ there exists a unique value $\alpha^{*}(\tilde{\pi} ; \gamma, m)$ such that

$$
\tilde{\pi}-\pi\left(\alpha^{*}(\tilde{\pi}, \gamma, m) ; \gamma, m\right)=0 .
$$

Thus the condition required for an interior solution for $\alpha$ to exist is that

$$
\begin{aligned}
\tilde{\pi} & >\frac{m}{E(\theta \mid \theta \geq m)} \\
\Rightarrow E(\theta \mid \theta \geq m) & >\frac{m}{\tilde{\pi}} .
\end{aligned}
$$


This last condition, which involves $E(\theta \mid \theta \geq m)$, imposes a restriction on the parameter space $\Gamma$ since a subset of the parameters belonging to $\gamma$ are those that characterize the distribution of $\theta$, and a subset of these parameters appear in the conditional expectation function $E(\theta \mid \theta \geq m)$. We have another condition that the estimated parameters must implicitly satisfy, of course, which is that $\rho V_{n}(m)<m$. We impose this condition directly by working with the parameterization

$$
\tilde{\gamma} \equiv\left(\rho V_{n}(m), \lambda, \eta, G\right)
$$

Under a lognormality assumption regarding $G$, the distribution of $\theta$ is completely characterized by the two parameters $\left(\mu, \sigma^{2}\right)$, so that the parameter space for $\tilde{\gamma}$ is given by

$$
\tilde{\Gamma}(m)=(-\infty, m) \times R_{+}^{2} \times R \times R_{+},
$$

since $\mu \in R$ and $\sigma^{2} \in R_{+}$. When we impose the additional condition that $E(\theta \mid \theta \geq m)>m / \tilde{\pi}$, under the lognormality assumption this implies that

$$
\exp \left(\mu+.5 \sigma^{2}\right) \frac{\tilde{\Phi}(z(m)-\sigma)}{\tilde{\Phi}(z(m))}>\frac{m}{\tilde{\pi}}
$$

where $z(m) \equiv(\ln (m)-\mu) / \sigma \cdot{ }^{12}$ Given values of $m$ and $\tilde{\pi}$, let the set of values of $\left(\mu, \sigma^{2}\right)$ that satisfy [20] be denoted by $\Omega(m, \tilde{\pi})$. Finally, define the parameter space

$$
\Gamma^{\prime}(m, \tilde{\pi})=\Gamma(m) \cap \Omega(m, \tilde{\pi}) .
$$

We form a concentrated likelihood function by substituting our estimator for $\alpha$ into the likelihood [15], which gives us

$$
\tilde{L}(\tilde{\gamma} ; \tilde{\pi}, m)=L\left(\tilde{\gamma}, \alpha^{*}(\tilde{\pi}, \tilde{\gamma}, m)\right)
$$

We have the following result.

Proposition 12 Assume that $\tilde{\gamma}^{0} \in \operatorname{int}\left(\Gamma^{\prime}(m, \tilde{\pi})\right)$ and $\alpha^{0} \in(0,1)$, where $\left(\tilde{\gamma}^{0}, \alpha^{0}\right)$ are the true population parameter values. Then the maximum likelihood estimators

$$
\begin{aligned}
\hat{\gamma} & =\arg \max _{\tilde{\gamma} \in \Gamma^{\prime}(m, \tilde{\pi})} \ln \tilde{L}(\tilde{\gamma} ; m, \tilde{\pi}) \\
\hat{\alpha} & =\alpha^{*}(\tilde{\pi}, \hat{\gamma}, m)
\end{aligned}
$$

converge in probability to $\tilde{\gamma}^{0}$ and $\alpha^{0}$ respectively. The asymptotic distribution of $\sqrt{N}\left(\hat{\gamma}-\tilde{\gamma}^{0}\right)$ is normal with mean 0 and covariance matrix $\Sigma_{\hat{\gamma}}$, which is of full rank. The asymptotic distribution of $\sqrt{N}\left(\hat{\alpha}-\alpha^{0}\right)$ is normal with mean 0 and variance $\sigma_{\hat{\alpha}}^{2}\left(\Sigma_{\hat{\gamma}}\right)$.

Proof: The unconcentrated likelihood function $L(\tilde{\gamma}, \alpha)$ satisfies standard regularity conditions for $\tilde{\gamma}^{0} \in \operatorname{int}(\tilde{\Gamma}(m))$ and $\alpha^{0} \in(0,1)$. In particular, the support of the distribution of the data is not a function of $\tilde{\gamma}^{0}$ and $\alpha^{0}$,though it is a function of the known constant $m$. The likelihood function is continuously differentiable in both $\tilde{\gamma}$ and $\alpha$. The implicit function $\alpha^{*}(\tilde{\pi}, \tilde{\gamma}, m)$ is continuously differentiable in $\tilde{\gamma}$, so the function $\ln L\left(\tilde{\gamma}, \alpha^{*}(\tilde{\pi}, \tilde{\gamma}, m)\right) \equiv \ln \tilde{L}(\tilde{\gamma} ; \tilde{\pi}, m)$ is continuously differentiable in $\gamma$ on the space $\Gamma^{\prime}(m, \tilde{\pi})$. Continuous differentiability of $\alpha^{*}$ is sufficient

\footnotetext{
${ }^{12}$ See Johnson and Kotz (1970, page 129).
} 
for $\operatorname{plim}_{\tilde{\gamma} \rightarrow \tilde{\gamma}^{0}} \alpha^{*}(\tilde{\pi}, \tilde{\gamma}, m)=\alpha^{0}$, and differentiability of $L(\tilde{\gamma}, \alpha)$ in both arguments is sufficient for $\operatorname{plim}_{\alpha \rightarrow \alpha^{0}} \operatorname{plim}_{N \rightarrow \infty} \arg \max _{\hat{\gamma} \in \tilde{\Gamma}(m)} \ln L(\tilde{\gamma}, \alpha)=\gamma^{0}$. Then $\operatorname{plim}_{N \rightarrow \infty} \alpha^{*}(\tilde{\pi}, \hat{\gamma}, m)$.

Since the concentrated $\log$ likelihood function $\ln \tilde{L}(\gamma ; \tilde{\pi}, m)$ satisfies all regularity conditions for asymptotic normality, the asymptotic distribution of $\sqrt{N}\left(\hat{\gamma}-\tilde{\gamma}^{0}\right)$ is $N\left(0, \Sigma_{\hat{\gamma}}\right)$, where

$$
\Sigma_{\hat{\gamma}}=\left(\left.\frac{\partial^{2} \ln \tilde{L}(\tilde{\gamma} ; \tilde{\pi}, m)}{\partial \tilde{\gamma} \partial \tilde{\gamma}^{\prime}}\right|_{\tilde{\gamma}=\hat{\gamma}}\right)^{-1} .
$$

The asymptotic covariance matrix of $\hat{\alpha}$ is derived using the delta method. The variance of $\hat{\alpha}$ is

$$
\operatorname{Var}(\hat{\alpha})=\left.\left.\frac{\partial \alpha^{*}(\tilde{\pi}, \tilde{\gamma}, m)}{\partial \tilde{\gamma}}\right|_{\tilde{\gamma}=\hat{\gamma}} ^{\prime} \Sigma_{\hat{\gamma}} \frac{\partial \alpha^{*}(\tilde{\pi}, \tilde{\gamma}, m)}{\partial \tilde{\gamma}}\right|_{\tilde{\gamma}=\hat{\gamma}} .
$$

As we shall see below, the use of wage share information from a firm or firms in conjunction with the CPS data allows us to obtain precise and credible estimates of all key model parameters. We do face a problem with this information when we attempt to estimate the model for subpopulations, however. Since we do not have wage and productivity information disaggregated by subpopulation, we are forced to assume that this ratio is the same across all subpopulation members. ${ }^{13}$ This is a strong assumption that probably largely accounts for the similarities we observe in the estimates of $\alpha$ across subpopulations.

\section{Empirical Results}

The data used contain information on 16 to 24 year olds [inclusive] from the September and October 1996 person files of the Outgoing Rotation Group (ORG) subsamples of the Current Population Survey (CPS). We focused attention on this age group since minimum wage workers are disproportionately young; our feeling was that if minimum wage effects on welfare were determined to be small for this group of participants, they are likely to be even more insignificant for older individuals. We focus on this particular period so that we can use the change in the minimum wage that occurred on September 30, 1996 as another potentially useful piece of identifying information. We also use data from these two months to test some implications of the model. Wages are measured in current period dollars and unemployment durations are measured in months.

Since our model is conditional on labor market participation, we have only used data for individuals who indicated that they were currently employed or were actively searching for employment. ${ }^{14}$ After imposing this selection criterion and excluding observations with missing data on key variables, we were left with sample sizes of 1867 in September and 1933 in October. In estimating the model a small number of additional observations with extremely low or high wage observations were excluded

\footnotetext{
${ }^{13}$ Another alternative would be to collect aggregate wage share information by firm, and to estimate the subpopulation-specific share using variability in subpopulation employment rates across firms. The use of such a procedure raises questions regarding the source of the variability in subpopulation employment rates across firms or industrial sectors and also faces the problem that few firms report wages as a separate item in their CSI.

${ }^{14}$ The standard CPS definition of unemployment is used, i.e., the individual is classified as unemployed if she or he has actively searched for a job within the past four weeks.
} 
Table 1 contains descriptive statistics for the two samples. We note that though general macroeconomic conditions were favorable in this period, the unemployment rate for this sample of young persons is much higher than the population unemployment rate (11.4 percent and 12.2 percent in September and October, respectively). This high rate is typically explained by the limited labor force attachment of large segments of this group, particularly those who are still attending school. In fact, we see that over 50 percent of the sample members indicate that they are currently enrolled in school. Due to this fact, the schooling attainment measure will be a lower bound for the completed schooling level of individuals still enrolled and the average and standard deviation of this measure should be interpreted accordingly.

While not directly used in the empirical analysis that follows, other characteristics of the sample that deserve mention concern the structure of the household and the survey design itself. The variable Self-Respond is an indicator variable that takes the value ' 1 ' when the household member in question has reported the information themselves. CPS interviewers gather information concerning all eligible household members from one respondent. When this respondent is reporting information about another member, it is natural to expect more inaccuracy in the report. About 26 percent of the data used were reported by a "proxy" respondent, which should be kept in mind in interpreting some of our results. The variable Child is an indicator that takes the value ' 1 ' when the subject is the child of the head of household. We see that about 40 percent of our sample members are children living in the homes of their parents. Such a living arrangement may indicate that the individual has a tenuous relationship to the market, or that she or he has been unsuccessful in labor market activities. In either case, it is probably much more likely that such an individual may be paid the minimum wage conditional upon employment.

We also report descriptive statistics regarding the dependent variables of the analysis, the ongoing unemployment spell duration (for the unemployed at the sampling time) and the hourly wage rate (for those employed at the sampling time). In terms of the continuing unemployment spell length, we see that there is a drop in the mean duration from September to October of approximately $1 / 2$ of a month. Recall that the rate of unemployment actually increased over this period. These observations are consistent with the negative employment effects of an increase in the minimum wage. An increase in the minimum wage will initiate a number of new unemployment spells as a result of the termination of low productivity matches (i.e., those for which $m \leq \theta<m^{\prime}$, where $m$ is the old and $m^{\prime}$ the new minimum wage rate). This type of effect could also account for the large decline in the standard deviation in the length of on-going unemployment spells observed between September and October.

The average wage of employed workers also increases between the two months, exhibiting a gain of about 13 cents. This is also consistent with implication of the model, though as we have pointed out in theory it is possible for an increase in the minimum wage to produce a decrease in the average wage rate of employed workers if there is a sufficiently large adverse impact on the value of search (although this is unlikely to be observed in practice). The standard deviation of hourly wages is approximately the same in the two months.

The last rows of Table 1 contain the proportion of the samples at each of a select number of hourly wage values. Under our assumption that the matching distribution is continuous, we should expect to observe significant amounts of "heaping" only at the minimum wage. ${ }^{15}$ Interestingly, we

\footnotetext{
${ }^{15}$ Because wages are reported in cents, even with a continuous matching distribution the probability that wage reports from two different individuals are identical is not zero. Clearly the significant amount of heaping at certain "focal" points is more than we would expect if this were the only factor involved.
} 
see that these 11 hourly wage values contain 59 percent of the wage observations in September and 62 percent in October. This seems to indicate that there is a significant amount of heaping in wage reports, though a definitive judgment can't be made without access to firm payroll records. We note that in terms of the size of the mass points, the respective minimum wages in the two months both rank fourth. The largest mass point in both months occurs at the value ' 5 ', which is reported for about $1 / 7$ of all employed workers. Other popular wage reports are ' 6 ' and ' 7 ', which together account for about $1 / 6$ of all wage reports in each month. Once again, while we cannot be sure that such reports are "erroneous," it is natural to suspect that this is the case.

Before assessing how problematic this "spurious" heaping might be for our analysis, let us consider the mass points in which we have a substantive interest. In September the federal minimum wage was $\$ 4.25$, and we see that this value was reported for almost 6 percent of the employed sample members. In September the proportion with a reported wage of $\$ 4.75$ was 2.7 percent, instead. The federal minimum wage was increased to $\$ 4.75$ by October, and in that month the proportion of the employed sample reporting that exact value jumped to 7 percent. It is interesting to note that the in October the proportion of observations for which a sub-minimum wage of $\$ 4.25$ was reported fell to 2.6 percent. These wage reports could be valid if the individual was in a job not covered by minimum wage legislation, or may simply reflect a lag in the adjustment of the individual's wage or a lack of knowledge by the respondent that the wage had been adjusted. These figures provide evidence that the change in the minimum wage had a direct impact on the wage distribution. According to our model, it may have had other significant indirect impacts as well.

We believe that the wage reports from the ORG-CPS samples are of high enough quality to enable reasonable estimates of primitive parameters to be obtained for essentially two reasons. First, the wage distributions seem to indicate a real presence of heaping at the minimum wage. While there is evidence of spurious heaping in wage reports, we note that neither of the values "4.25" or " $4.75 "$ are particularly good candidates for "focal point" status. Second, as regards the probability masses at the focal points, inspection of the wage histograms in Figure 1 reveal that the heaping pattern is fairly regular. That is, the mass at each focal point is roughly proportional to the total mass in the "neighborhood" around it. Because of this, it seems reasonable to infer that values "close" to each focal point have been shifted to it. ${ }^{16}$ Our hope is that whatever biases may be produced by the heaping at focal points may be second-order.

In estimating the model our main focus will be the September 1996 sample. This is due to the fact that our model is premised on there being a stationary environment, and the environment immediately following a change in the minimum wage is not stationary due to the time required to move from the stationary equilibrium associated with the minimum wage of $m$ to the one associated with the minimum wage of $m^{\prime} .{ }^{17}$ We will use the October sample to check on various implications of our model toward the end of this section.

Our first attempt to estimate values of primitive parameters imposes a variety of normalizations (i.e., identifying restrictions) on the model. While the model is theoretically identified under any of

\footnotetext{
${ }^{16}$ While there exist methods for "redistributing" mass away from focal points to other points in their neighborhoods, we have not attempted such an adjustment here. Without access to "true" wage measures that could be used for validation, any measurement model is ad hoc and may induce more bias than it removes.

${ }^{17}$ While we assume that the wage rate would be adjusted immediately upon enactment of the minimum wage, the distribution of on-going unemployment spells would not be negative exponential in October due to the mixture of acceptance wages (4.25 and 4.75) which most spells would have been generated by. In contrast, all of the unemployment spells in September would have been generated by the minimum wage of 4.25 and the constant primitive parameters.
} 
the distributional assumptions we have made, as we will see below identification is tenuous at best. In Table 2 we report estimates of the model assuming that the matching distribution is lognormal, Gamma, or $\chi^{2}$ (the $\chi^{2}$ is a Gamma distribution with 2 degrees of freedom). Because the $\chi^{2}$ is a special case of a Gamma, we also report estimates from a specification in which $G$ is assumed to be $\chi^{2}$ but $\alpha$ is estimated as a free parameter. In all other specifications reported in Table 1 we have restricted $\alpha$ to equal 0.5 - the symmetric Nash bargaining case.

The estimates in Table 2 are obtained using two slightly different samples. The sample used to compute the estimates of parameters in the first panel simply excluded all employed sample members who were paid less than $\$ 4.25$ in September 1996 . The presumption in this case was that these individuals were operating in a different labor market than the formal one modeled in this paper. The sample used to compute the estimates presented in the second panel included all of these individuals instead of deleting them, but rounded all of their wage reports up to the minimum wage of $\$ 4.25$. Clearly this results in a much larger mass point at the minimum wage rate. A possible rationale for this procedure is that these respondents have systematically underreported their minimum wage salaries because of uncertainty regarding its level, or are employed at jobs that include other forms of compensation, which taken together would equal (or may exceed) the mandated minimum wage. We have reported results using both samples in an effort to determine the degree of sensitivity of our behavioral inferences to the handling of this type of "measurement error."

In the first column of Table 2 we present model estimates under the assumption that the matching distribution is lognormal and that Nash bargaining is symmetric (i.e., $\alpha=.5$ ). For this estimation exercise we have pooled all observations (that is, no matter what their differences in terms of observable characteristics) and assumed that all sample members operate within a homogeneous environment. Estimates in the top panel indicate that the average ln match value draw in the population is 2.099 with a standard deviation of .486 (the implied mean of $\theta$ in levels is 9.181). The average time between contacts for searchers is $1 / .327$, slightly more than 3 months. Since the acceptance value for a match draw under a binding minimum wage is $m$, the estimated average length of an unemployment spell is $1 /(\widehat{\lambda \tilde{G}(m)})=1 / .298=3.356$ months, essentially identical to the sample average. The estimated dissolution rate of jobs is 0.04 , implying that the average length of a job is 25 months. Such an estimate is reasonable considering the youth of the sample members and the degree of turnover in the U.S. labor market. Finally, the "implicit" reservation wage is estimated to be 3.621. In performing the estimation this value is always constrained to be less than the minimum wage in effect at the time.

The second column in the top panel of Table 2 contains estimates under the Gamma specification of the matching distribution where once again symmetric Nash bargaining has been assumed. There are few changes in the estimated values of any of the rate parameters. The estimates of the parameters characterizing $G$ change markedly of course, since they are not comparable across the two specifications. The estimate of the implicit reservation wage is higher than under the lognormal specification, though not markedly so. Since the same number of parameters have been estimated, the likelihood function value indicates a somewhat worse fit under the Gamma than under the lognormal specification.

The last two columns of the top panel report estimates under the $\chi^{2}$ assumption on $G$ with and without the symmetric Nash bargaining restriction. When $\alpha$ is restricted to equal 0.5 the rate parameters are little changed with respect to those reported in the first two columns. Naturally, the estimates of the parameters characterizing $G$ are different than those reported in columns 1 and 
2. The specification in column 3 is in fact a special case of the one reported in column 2 , and we see that the estimate of the scale parameter $\zeta_{2}$ reported in column 2 is significantly different from the value ' 2 ' associated with the $\chi^{2}$. Given the restriction that $\alpha=.5$ we would tend to reject the $\chi^{2}$ restriction that $\zeta_{2}=2$ and preference would be given to the unrestricted Gamma specification.

Because the parameter $\zeta_{2}$ is a scale parameter, and it is the presence of a scale parameter that significantly complicates the estimation of the bargaining power parameter $\alpha$, we have attempted to estimate $\alpha$ under the $\chi^{2}$ assumption. The results appear in the fourth column. We notice that there are substantial differences between the estimates in columns 3 and 4 as a result of treating $\alpha$ as a free parameter. Most interestingly, we note that the estimate of $\alpha$ is .708, which indicates that employees have substantially more bargaining power than employers. This result is surprising, particularly given the fact that the sample is so young and contains many students. Though the estimate of $\alpha$ is relatively precise, the reason is the distributional assumption we have made in column 4 .

The bottom panel of Table 2 reports estimates of the same specifications using the sample that includes all employed individuals who reported a wage less than the minimum; all such individuals were assigned a wage equal to the minimum. The estimates for each of the specifications are quite similar between the top and bottom panels with the exception of the implicit reservation wage. This is to be expected since by the construction of the sample used to produce the estimates in the lower panel we have created many more observations at the minimum wage. In order to increase the amount of heaping at that value the reservation wage is reduced. We conclude that use of estimates from either sample is not likely to greatly change the implications of the policy experiments we conduct below.

In Section 4.3 we described an estimator that utilized the same CPS data that has been employed to this point and one additional piece of demand side information, the ratio of total wages paid to firm revenues. It goes without saying that our model is highly stylized, and that in reality there is no single value of this ratio when looking across firms in the economy at any point in time. We have opted to use the value (or a reasonable approximation to it) from one large U.S. corporation, McDonald's. This firm has been selected for a number of reasons. First, it is the largest private sector employer in the U.S., so that the law of large numbers results which we relied upon in Section 4.5 are appropriate. Second, this firm has a disproportionate number of employees from the age group in which we are interested, those between the ages of 16 and 24. Moreover, a large number of its employees are paid at or near minimum wage level. Third, McDonald's is one of the few large employers in this sector that reports sufficiently disaggregated information in its Consolidated Statement of Income (CSI) to allow computation of the number in which we are interested.

In obtaining a value for the wage share at McDonald's we have used information from the company's 1996 CSI, which covers a period that includes the survey month of the CPS we are using (September 1996). For purposes of homogeneity we have restricted attention to revenues and costs associated with restaurants owned by the company (i.e., we have excluded franchises). We computed our value of "net" revenue by subtracting "costs of food and packaging" plus "occupancy and other operating expenses" from total sales by Company-operated restaurants." These two expense items are essentially treated as fixed costs. The numerator of the ratio is given by "payroll and other employee benefits." We found the value of this ratio to be 0.576 in 1996 . It is worthwhile to note that the ratio seems to be relatively stable over time. For example, using the same computational method the ratio was equal to 0.568 in 1995 and 0.560 in 1994. 
Table 3 contains estimates of the model with the profit information included. The first column of the top panel contains the estimates for the entire sample with those employed at a wage less than 4.25 excluded. The addition of the demand side information has resulted in virtually no change in the sampling distribution associated with $\lambda$ and $\eta$, as was to be expected from the identification discussion provided above. Conversely, we notice a very large change in the estimates of the matching distribution parameters and the bargaining power parameter. The point estimates of $\mu$ and $\sigma$ are 2.278 and 0.521 , respectively, and their standard errors of 0.029 and 0.018 indicate that they are precisely estimated. The point estimate of the implicit reservation wage is 3.685 and its estimated standard error is 0.082 .

Of most interest to us is the estimate of the bargaining power parameter. The point estimate of $\alpha$ is 0.378 and the estimated standard error of $\alpha$ is $0.008 .{ }^{18}$ With this level of precision, it is straightforward to reject the null hypothesis of symmetric Nash bargaining. The estimate clearly indicates that this class of worker is at a disadvantage with respect to extracting match surplus. The use of the profit information adds a tremendous amount of information regarding the estimation of the size of the pie to be divided and has resulted in what we consider a very reasonable estimate. ${ }^{19}$

The other columns in the top panel of Table 3 demonstrate the relative stability of the $\alpha$ estimates across demographic groups. As was discussed in the Section 4.3, this near constancy is largely an artifact of our estimation procedure. Since productivity and wage information is not available by demographic group in the company's CSI we have had to assume that the same ratio applied to all groups. Since this is the key piece of identifying information in determining $\hat{\alpha}$, near constancy of these estimates is almost guaranteed. To our knowledge there exists no individual level data set in existence that could be used to construct these values. ${ }^{20}$

Even if the group-specific values of $\hat{\alpha}$ are relatively similar, because of the high degree of precision with which they are estimated there do exist statistically significant differences between them. For example, youths who are black or Hispanic are estimated to have a slightly lower level of bargaining power than others $(0.357$ versus 0.382$)$. They also have a slightly lower estimated value of the mean of the logarithm of the match value, but have a slightly higher standard deviation of log match values. When we divide the sample along gender lines, we find that males are estimated to have a slightly higher degree of bargaining power than females, as well as having higher values of $\mu$ and $\sigma$.

The estimates in the bottom panel of Table 3 are broadly consistent with those in the top panel. Once again, because the size of the mass point at $m$ has increased substantially we see that the estimated implicit reservation wage is significantly lower than in the top panel for each demographic group and the aggregate. Estimates of $\alpha$ tend to be slightly higher than in the top panel, while estimates of rate parameters and $\mu$ and $\sigma$ are quite similar in the two panels.

In Table 4 we use the model to directly determine whether or not the minimum wage increase that occurred at the end of September 1996 was beneficial in the sense of our first welfare measure, the value of unemployed search. In this analysis we only estimate the model for the aggregate, and

\footnotetext{
${ }^{18}$ It is interesting to note that Cahuc et al (2002) and Yashiv (2003) report much lower estimates of the worker's bargaining power, though both use very different data sources and somewhat different equilibrium models. I find the high profit rates implied by such low bargaining power estimates to be problematic.

${ }^{19}$ The mode of our prior on $\alpha$ was 0.4 . While some colleagues expressed the opinion that $\alpha$ should be near 0 for this group of searchers, such a value would imply an unreasonable profit rate for firms like McDonalds as well as a wage distribution with too little variance.

${ }^{20}$ Our highly stylized model posits that firm productivity is the sum of individual productivities, which is not likely to be the case in practice. This implies that one would have problems in assigning productivity levels to subsets of employees in practice, even if group wages could be unambiguously defined.
} 
we begin by combining the September 1996 ORG-CPS sample with the analogous one for October 1996. Given the sample design these two surveys do not include the same individuals. At the top of the columns of Table 4 the samples are the labels $S 1$ and $S 2$. The sample $S 1$ includes all unemployed individuals in September and October as well as those who were employed in September at an hourly wage no less than $\$ 4.25$ and those employed in October at an hourly wage no less than $\$ 4.75$. $S 2$ includes all of those in $S 1$ plus all employees who were excluded by the wage selection criteria used in extracting $S 1$. In $S 2$, all September employees who reported a wage less than $\$ 4.25$ were assigned a wage of $\$ 4.25$ and in October all those employees who reported a wage less than $\$ 4.75$ were assigned a wage of $\$ 4.75$.

Before turning to the results we must present a word of caution in terms of the interpretation of the estimates. Strictly speaking, the consistency of our estimator in this case depends on the search environment remaining stationary in the wake of the minimum wage change, so that all "primitive" parameters assume the same values in September and October 1996. ${ }^{21}$ There are a number of reasons why this assumption may be questionable; we shall consider a limited test of it below.

For our inferences to be valid requires constancy of environmental parameters, however we do not have to make any assumptions regarding whether the impending minimum wage change in October was anticipated by searchers and employees in September as long as a certain restriction on the minimum wages and the implicit reservation values is satisfied. Since the minimum wage bill was signed into law a few months before its actual implementation date, ${ }^{22}$ we can assume that individuals knew of the change that was to occur prior to its occurrence. This knowledge will have no effect on behavior as long as the implicit reservation wage after the new minimum wage is put into effect is no larger than the lower minimum wage. In terms of our application, we require $\rho V_{n}(4.75)<4.25$. If this is the case, then even under the old minimum wage match values in the interval $[4.25,4.75)$ will be accepted since working at the wage associated with such a $\theta$ given $m=4.25$ has a higher value than waiting to search under the new minimum wage. Point estimates from the model indicate that this restriction is not unreasonable, particularly when using $S 2$. As a result, we will assume that all offers in the interval $[4.25,4.75)$ remain acceptable to searchers in September even given their knowledge that acceptance of such matches implies reentry into the unemployment state on October 1.

The results in Table 4 provide some support for the claim that the increase in the minimum wage on October 1 resulted in a welfare increase, or at least not a decrease, under our welfare criterion $W C 1$. Using $S 1$, we find that $W C 1$ increased by a statistically significant 10.7 percent, while using $S 2 W C 1$ increased by a statistically insignificant 3.5 percent. It is of interest to note that in a companion paper that utilizes nonparametric methods (Flinn, 2003) I find weak or little support for the claim that the minimum wage increase to $\$ 4.75$ resulted in a $W C 1$ gain for this population of labor market participants.

\footnotetext{
${ }^{21}$ The assumption that the primitive parameters are invariant with respect to a policy change does not imply that the distribution of unemployment durations in October corrsponded to the steady state distribution of on-going spells of unemployment at the new minimum wage $m^{\prime}=\$ 4.75$. The unemployment distribution in October carries over searchers from September but decreases their exit rate to $\lambda \tilde{G}(4.75)$ from $\lambda \tilde{G}(4.25)$. In addition, those employed in September at a match value between 4.25 and 4.75 are added to the unemployment pool, thus creating a large proportion of unemployment spells at low duration levels in October. All of these effects have been incorporated into the likelihood function for the September and October data.

${ }^{22}$ On August 20, 1996, President Clinton signed the bill (HR 3448) containing the minimum wage increase of. 50 cents on October 1, 1996 and an additional 40 cents on September 1, 1997. Even before the date when the increases were officially signed into law it is reasonable to assume that an increase was widely anticipated by the general public.
} 
The validity of our direct estimate of the welfare level of labor market participants under two different minimum wage laws relies heavily on our assumption that the primitive parameters of the model were constant in the two policy environments. This assumption may be suspect for a number of reasons, even if we have looked at labor market outcomes separated by only one month in time. We will now consider one possible violation of this assumption, one that concerns the rate of encounters between searchers and firms. This rate, $\lambda$, is a focus of interest of many macroeconomic search-based models of the labor market (see, e.g., Pissarides (2000)), where it is commonly assumed that $\lambda=\lambda(u, v)$, with $u$ representing the number of unemployed searchers and $v$ the number of vacancies. We might imagine that an increase in the minimum wage would induce more individuals to enter the market as searchers, while simultaneously decreasing the number of firms posting vacancies. Under this line of argument, it is reasonable to expect that the rate of contacts between searchers and firms with vacancies would decrease with an increase in $m$, at least if the minimum wage increase resulted in a higher welfare level (in equilibrium) for workers and a lower welfare level for firms.

The results in Table 5 are consistent with this intuition. We find evidence that the increased minimum wage in October was associated with higher welfare levels on the supply side of the market, as before, and that the rate of contacts between searchers and firms was lower under the new minimum wage. While it appears that at least one "primitive" parameter was not in fact invariant with respect to the policy change, this violation of our modeling assumption has no substantive impact on our assessment of the welfare impact of the change in the minimum wage law. This is due to the fact that there is no covariance between the estimators of $\rho V_{n}$ and $\lambda$. In fact, there is no covariance between $\widehat{\rho V_{n}}$ and the estimator of either rate parameter. Thus shifts in these parameters with the minimum wage will have no effect on our estimate of the welfare effect of the minimum wage increase.

Misspecification of the model in the sense of having "primitive" parameters that are not policy invariant will create problems for the policy experiments presented immediately below. In particular, if the rate of contacts between workers and firms $(\lambda)$ is decreasing in $m$, then the optimal minimum wage rates we find will be too high, in general. The only way to get around this problem would be to build and estimate a general equilibrium model in which $\lambda$ is determined endogenously. While one could follow the lead of Pissarides (2000) and model the firm vacancy decision, we feel that this would not be enough for this particular application. A realistic model would also have to explicitly model the participation decision of young people, many of whom are in enrolled in school. The creation of such a model is beyond the scope of the current exercise and is left for future research.

\section{Policy Experiments}

The results of our policy experiments, all of which only involve shifts in the minimum wage, are summarized in Figures 5-7 and in Table 6. For all of the experiments point estimates from the bottom panel of Table 3 have been used. We begin by discussing the results presented in Figure 5 and Table 6, which use the estimates for the total sample (those that appear in the first column of Table 3).

Figure 5.a plots the implicit reservation wage as a function of the minimum wage rate. We notice that this function is single-peaked, and has its maximum at the value of $\$ 8.46$, which is 
almost twice the minimum wage rate of $\$ 4.25$ that was in effect in September of $1996 .{ }^{23}$ While this is the "optimal" minimum wage rate given our estimates of the primitive parameters, we see from the function that any minimum wage greater than $\$ 4.25$ but less than $\$ 11.23$ would have resulted in a welfare gain under $W C 1$. The parameters primarily responsible for this large value of $m^{*}$ are the bargaining power $(\alpha=0.409)$ and the rate of contacts between firms and searchers $(\lambda=0.298)$, which are assumed invariant with respect to $m$.

In Figure 5.b we have plotted the proportion of labor market participants who experience a welfare gain when the minimum wage is changed from $\$ 4.25$ to $\mathrm{m}$. The actual experiment utilizes the steady state distribution of $\theta$ induced by the minimum wage $m$, and using this distribution we compute the proportion of population members (i.e., values of $\theta)$ for which $S\left(\theta ; m^{\prime}\right) \geq S(\theta ; m)$. We see from the Figure 5.b that, starting from $m=\$ 4.25$, small changes in $m$ result in welfare gains for most market participants, with only a few of those who lose their jobs due to the increase becoming worse off. When the minimum wage increases to $\$ 5.20$, all agents in the market are better off under the change. The proportion benefitting from a minimum wage change only begins to decline (from 1.0) when the new minimum wage reaches $\$ 10.21$. If the minimum wage changed from $\$ 4.25$ to $\$ 10.90,50$ percent of the population would gain from this large increase. We loosely characterize this value as the one that would be selected by the median voter.

Figure 5.c contains a plot of the average steady state labor market value as a function of the minimum wage. The single-peaked shape that we saw in Figure 5.a is again present, though the $E_{S S} S(\theta ; m)$ function appears a bit flatter than $V_{n}(m)$. The optimal minimum wage using this criterion $(W C 3)$ is $\$ 9.02$, which is even larger than the optimal minimum wage indicated under $W C 1$.

Table 6 summarizes some of these results, and also presents the steady state unemployment rate associated with the various "optimal" minimum wages. When the minimum wage is increased to $\$ 8.46$, as is indicated under $W C 1$, the steady state unemployment rate is increases by 46 percent. When the minimum wage is set at $\$ 10.90$, the SS unemployment rate exactly doubles. These large increases underscore the fact that the unemployment rate is only one component of the total welfare impact of a minimum wage increase. Put differently, "optimal" increases in minimum wage may engender large increases in the steady state unemployment rate of impacted groups.

The results in Table 6 also indicate that when the minimum wage is set according to $W C 1$ or $W C 3$, the welfare gains in the relevant measures are substantial. In particular, when the minimum wage is changed from $\$ 4.25$ to $\$ 8.46$, there is a 17 percent increase in the value of search. When the minimum wage is changed from $\$ 4.25$ to $\$ 9.02$, there is a 20.7 percent increase in the expected steady state value of labor market participation.

While there is some variation of mandated minimum wages by state, all residents of a given geographic area working in the same industry (since some industries have exemptions from "standard" minimum wage regulation) are subject to the same minimum wage. Nonetheless, demographic groups may be differentially impacted by a common minimum wage change due to the characteristics of the submarkets in which they operate.

Using group-specific estimates from the bottom panel of Table 3, in Figure 6 we have plotted $\rho$ times the value of unemployed search as a function of the minimum wage. Figures 6.a and 6.b

\footnotetext{
${ }^{23}$ While we have not been able to prove that $V_{n}(m)$ is always a single-peaked function of $m$ for any matching distribution $G$, in working with the lognormal distribution we have always found this to be the case. For relatively low values of $\alpha$, such as those in Table 3, the single-peaked form of Figure 5.a is found. For relatively large values of $\alpha$, the function $V_{n}(m)$ is monotonically decreasing in $m$ as soon as $m$ becomes binding. In this case the "peak" is the constant value of $V_{n}(0)$ that extends from $m=0$ through $m=\rho V_{n}(0)$.
} 
plot this function for the subsamples of males and females. We see the same single-peaked shape that characterized the function derived from the estimates for the total sample. We find that the optimal minimum wage under $W C 1$ is $\$ 8.93$ for males and $\$ 8.04$ for females. While there is a significant difference between these two values, we do note that either group would experience an increase in its value of search under the other group's optimal minimum wage (when beginning from $m=\$ 4.25$ ). When we break the sample along racial-ethnic lines we find a similar result. The optimal minimum wage for blacks and Hispanics would be $\$ 8.75$ under this criterion in comparison with a minimum wage of $\$ 8.37$ for the white subpopulation. This is a reasonably small difference and, once again, either group would be far better off under the optimal minimum wage of the other than at the value $m=\$ 4.25$.

Figure 7 contains plots of the proportions of the various subpopulations that experience a welfare gain when the minimum wage is increased from $\$ 4.25$ to $\mathrm{m}^{\prime}$. Comparing males and females, we see that there is a large difference between the "median voter" value of $m$ in the two labor markets. When the minimum wage moves from $\$ 4.25$ to $\$ 11.64$, half of the male labor market participants would experience a welfare gain. For females that point comes at a minimum wage value of $\$ 10.25$. In fact, if the minimum wage were increased to $\$ 11.64$ from $\$ 4.25$, all females would experience a welfare loss. The situation is similar, though a bit less extreme, when we consider the contrast between white nonhispanics and the group composed of blacks and Hispanics. The "median voter" value for white nonhispanics is $\$ 10.62$, while it is equal to $\$ 11.88$ for blacks and Hispanics. While some nonhispanic whites would still gain from a minimum wage of $\$ 11.88$, the proportion doing so is only about 22 percent. It is important to note that all groups experience Pareto improvements when the minimum wage increases from $\$ 4.25$ to any $m^{\prime}$ (roughly) in the interval $[6,10]$.

Figure 8 contains plots of the expected steady state value of labor market participation as a function of the minimum wage for the various subpopulations. The patterns we see are roughly consistent with those exhibited in Figure 6; that is, each curve is single-peaked, though with different degrees of curvature. A minimum wage in the range $[6,10]$ would clearly produce welfare gains under this metric for any of the subpopulations.

It may be instructive to compare the results of these experiments with the estimates presented in Table 4. Recall that in that table we directly estimated the welfare gain (in terms of $W C 1$ ) associated with moving from $m=\$ 4.25$ to $m^{\prime}=\$ 4.75$. We found only weak evidence that $V_{n}\left(m^{\prime}\right)>V_{n}(m)$. On the other hand, our experimental evidence indicates that there are potentially extremely large payoffs to searchers from significant increases in the minimum wage rate. There is no contradiction here when we recognize that small increases in the minimum wage are not likely to lead to significant welfare improvements, based on the estimated values of the primitive parameters. Our model indicates that substantial welfare impacts can only be observed in response to large changes in $m$, which is something that has never occurred historically.

\section{Conclusion}

We have formulated a simple partial equilibrium model of wage determination that carries implications for the effects of minimum wage changes on accepted wage distributions and unemployment spell lengths broadly in accord with the findings of other researchers who have worked with disaggregated data. By simply appending a side constraint to the standard Nash bargaining problem, we were able to generate accepted wage distributions with a mass point at the minimum wage and a continuous density of wages to the right of $m$. The model is parsimoniously specified in that 
the equilibrium wage and unemployment spell distributions are characterized in terms of seven primitive parameters.

Using the model, we defined several welfare criteria, though the focus of interest was mainly on the value of unemployed search. Besides being the ex ante value of the labor market career, this welfare criterion is also Rawlsian in the sense that unemployed searchers have the lowest welfare level over all labor market participants. It also has the advantage of being a scalar measure and so serves as a convenient welfare index. We showed, using this measure and others, that the impact of minimum wage changes on the welfare of searchers and labor suppliers was impossible to determine without knowledge of the primitive parameters of the model. We thus set out to estimate these parameters using the limited amount of information available from the Current Population Survey.

The welfare impacts of minimum wages hinge most critically on the size of the bargaining power parameter $\alpha$; unfortunately, as a number of previous researchers have discovered, this is an exceedingly difficult parameter to identify. We demonstrate formally why this is the case. In essence, the problem is that the wage is a linear mapping from the match distribution space, and hence parameters that characterize this mapping (most notably $\alpha$ ) are indistinguishable from any unknown location and scale parameters characterizing $G$, the distribution function of the match value. This problem is unsolvable without imposing unrealistic restrictions on $G$ a priori or through the use of arbitrary exclusion restrictions. Neither seem an attractive route to pursue.

We demonstrated that by using a very limited amount of information from the demand side of the market it was possible to obtain extremely precise estimates of $\alpha$ and other primitive parameters. By using some information from the demand side, we are essentially able to fix the size of the "pie" to be divided and hence can recover parameters that determine the share going to workers in an efficient manner. The estimates of $\alpha$ that we obtained allowed us to decisively reject the null hypothesis of symmetric Nash bargaining. Our estimates of $\alpha$ for the aggregate and across various subpopulations were in the neighborhood of 0.4. Along with the estimates of the other primitive parameters, this created the possibility of considerable welfare gains (to the supply side of the market) from large increases in the minimum wage. Though our model is likely to over-estimate the size of the "optimal" minimum wage, we found that an approximate doubling of the mandated minimum wage in September 1996 was indicated using a variety of welfare measures.

While we have shown that large increases in minimum wages can lead to substantial increases in the welfare of impacted populations, clearly there are a number of caveats to be added. We presented evidence that the rate of contacts between workers and firms does not appear to be invariant with respect to minimum wage changes, i.e., it is not properly considered a primitive parameter. It appears that the rate of contacts decreases after a minimum wage change, which is consistent with what would be expected when using a Pissarides-style matching function $\lambda(u, v)$ when the number of unemployed searchers $(u)$ increases and the number of vacancies $(v)$ declines. While our data do not contain the intertermporal variability required to estimate the function $\lambda(\cdot, \cdot)$, it would be an important extension of our modeling framework to allow for changes in $\lambda$ resulting from firms reducing their vacancy creation rate (due to decreased expected profits) and entry into the labor market by individuals attracted by the increased value of unemployed search. These generalizations should result in reductions in the "optimal" minimum wage rate.

Another major omission is on-the-job (OTJ) search. It is well-known that over one-half of all job spells terminate with the direct transition into another job; the proportion is even higher for young labor market participants. In terms of the impact of OTJ in our model, it is not difficult to show that when OTJ search is possible, and firms compete for a worker by making alternating offers 
until the firm with the lowest match value offers a wage equal to that value (see Dey and Flinn (2003) or Postel-Vinay and Robin (2002) for a different but related renegotiation method), only the first job in any consecutive sequence of jobs can possibly pay the minimum wage. As a result, workers will spend a lower proportion of their labor market careers being paid the minimum wage, which leads us to suspect that minimum wages will have less impact on the value of labor market careers than in the no OTJ search case analyzed here. To analyze this case requires information not available in the CPS, so the final verdict of the impact of minimum wages in the presence of OTJ search must await a fresh analysis using different data.

Let us conclude by considering the larger issue of the effectiveness of the minimum wage as policy instrument. The minimum wage is a crude, blunt tool, the use of which unquestionably creates deadweight losses. As we saw through examples and simulations based on estimated parameters, large increases in unemployment can result from significant increases in the minimum wage. On the positive side, the minimum wage is easy to understand, implement, and enforce. Its role in the asymmetric Nash bargaining model was particularly clear. Given low bargaining power, which in conjunction with other unfavorable values of labor market parameters gives searchers low threat points, the worker's share of the match value is small. Without the ability to directly impact the bargaining power parameter itself, the government is at least able to set the minimum wage as a coordination device for searchers in this environment. Because bargaining is bilateral, no individual searcher has the wherewithal to hold out for a higher share of the surplus in any given match. The government's role is to provide the discipline, through setting a lower bound on wage contracts, that enables labor market participants to improve their bargaining position vis-a-vis employers. This can be an effective tool for a government that wants to redistribute welfare, though it comes with a number of costs, the most important of which is increased unemployment. 
Table 1

Descriptive Statistics

September and October 1996 CPS-ORG

Individuals Aged 16-24

(Standard Deviations in Parentheses)

\begin{tabular}{|c|c|c|}
\hline Characteristics & September & October \\
\hline$N$ & 1867 & 1933 \\
\hline$N_{u} / N$ & 0.114 & 0.122 \\
\hline Schooling & $\begin{array}{l}12.318 \\
(1.801)\end{array}$ & $\begin{array}{l}12.309 \\
(1.896)\end{array}$ \\
\hline Female & 0.488 & 0.486 \\
\hline Black & 0.092 & 0.113 \\
\hline Hispanic & 0.106 & 0.121 \\
\hline Enrolled & 0.557 & 0.538 \\
\hline Self-Respond & 0.738 & 0.738 \\
\hline Child & 0.403 & 0.410 \\
\hline$t$ & $\begin{array}{c}3.361 \\
(5.023)\end{array}$ & $\begin{array}{c}2.803 \\
(3.943)\end{array}$ \\
\hline$w$ & $\begin{array}{c}6.523 \\
(2.425)\end{array}$ & $\begin{array}{c}6.654 \\
(2.456)\end{array}$ \\
\hline$\chi[w=4.25 \mid e]$ & 0.059 & 0.026 \\
\hline$\chi[w=4.50 \mid e]$ & 0.022 & 0.021 \\
\hline$\chi[w=4.75 \mid e]$ & 0.027 & 0.070 \\
\hline$\chi[w=5.00 \mid e]$ & 0.141 & 0.133 \\
\hline$\chi[w=5.25 \mid e]$ & 0.029 & 0.030 \\
\hline$\chi[w=5.50 \mid e]$ & 0.039 & 0.057 \\
\hline$\chi[w=6.00 \mid e]$ & 0.095 & 0.099 \\
\hline$\chi[w=7.00 \mid e]$ & 0.070 & 0.074 \\
\hline$\chi[w=8.00 \mid e]$ & 0.054 & 0.045 \\
\hline$\chi[w=9.00 \mid e]$ & 0.022 & 0.030 \\
\hline$\chi[w=10.00 \mid e]$ & 0.029 & 0.036 \\
\hline al at These Values & 0.587 & 0.621 \\
\hline
\end{tabular}




\section{Table 2}

\section{Model Estimates with Normalizations September 1996 CPS-ORG}

\begin{tabular}{|c|c|c|c|c|}
\hline \multirow[b]{2}{*}{ Parameter } & \multicolumn{4}{|c|}{ Matching Distribution } \\
\hline & Log Normal & Gamma & $\chi^{2}$ & $\chi^{2}$ \\
\hline \multirow{3}{*}{$\lambda$} & \multicolumn{4}{|c|}{ Excluding All $w<m(N=1781)$} \\
\hline & 0.327 & 0.391 & 0.332 & 0.428 \\
\hline & $(0.023)$ & $(0.031)$ & $(0.023)$ & $(0.044)$ \\
\hline \multirow[t]{2}{*}{$\eta$} & 0.040 & 0.040 & 0.040 & 0.040 \\
\hline & $(0.004)$ & $(0.004)$ & $(0.004)$ & $(0.004)$ \\
\hline \multirow[t]{2}{*}{$\zeta_{1}$} & 2.099 & 2.633 & 4.519 & 3.231 \\
\hline & $(0.028)$ & $(0.264)$ & $(0.093)$ & $(0.241)$ \\
\hline \multirow[t]{2}{*}{$\zeta_{2}$} & 0.486 & 3.036 & 2 & 2 \\
\hline & $(0.017)$ & $(0.216)$ & - & - \\
\hline \multirow[t]{2}{*}{$\rho V_{n}(m)$} & 3.621 & 3.767 & 3.526 & 3.444 \\
\hline & $(0.087)$ & $(0.060)$ & $(0.088)$ & $(0.123)$ \\
\hline \multirow[t]{2}{*}{$\alpha$} & 0.500 & 0.500 & 0.500 & 0.708 \\
\hline & - & - & - & $(0.047)$ \\
\hline \multirow[t]{2}{*}{$\ln L$} & -4332.525 & -4345.174 & -4366.605 & -4347.190 \\
\hline & \multicolumn{4}{|c|}{ Including All $w<m(N=1859)$} \\
\hline \multirow[t]{2}{*}{$\lambda$} & 0.320 & 0.382 & 0.324 & 0.417 \\
\hline & $(0.023)$ & $(0.031)$ & $(0.023)$ & $(0.043)$ \\
\hline \multirow[t]{2}{*}{$\eta$} & 0.038 & 0.038 & 0.038 & 0.038 \\
\hline & $(0.004)$ & $(0.004)$ & $(0.004)$ & $(0.004)$ \\
\hline \multirow[t]{2}{*}{$\zeta_{1}$} & 2.147 & 2.723 & 4.757 & 3.319 \\
\hline & $(0.034)$ & $(0.290)$ & $(0.115)$ & $(0.263)$ \\
\hline \multirow[t]{2}{*}{$\zeta_{2}$} & 0.472 & 3.014 & 2 & 2 \\
\hline & $(0.018)$ & $(0.220)$ & - & - \\
\hline \multirow[t]{2}{*}{$\rho V_{n}(m)$} & 3.082 & 3.385 & 2.909 & 2.823 \\
\hline & $(0.151)$ & $(0.093)$ & $(0.138)$ & $(0.192)$ \\
\hline \multirow[t]{2}{*}{$\alpha$} & 0.500 & 0.500 & 0.500 & 0.704 \\
\hline & & & & $(0.048)$ \\
\hline $\ln L$ & -4537.371 & -4549.095 & -4567.917 & -4550.898 \\
\hline
\end{tabular}


Table 3

Model Estimates with Profit Information

September 1996 CPS-ORG

\begin{tabular}{|c|c|c|c|c|c|}
\hline \multirow{3}{*}{ Parameter } & \multicolumn{5}{|c|}{ Demographic Group } \\
\hline & All & Males & Females & White Non-H & Black or $\mathrm{H}$ \\
\hline & \multicolumn{5}{|c|}{ Excluding All $w<m$} \\
\hline \multirow[t]{2}{*}{$\lambda$} & 0.315 & 0.271 & 0.384 & 0.336 & 0.293 \\
\hline & $(0.022)$ & $(0.026)$ & $(0.039)$ & $(0.029)$ & $(0.038)$ \\
\hline \multirow[t]{2}{*}{$\eta$} & 0.040 & 0.036 & 0.048 & 0.035 & 0.065 \\
\hline & $(0.004)$ & $(0.005)$ & $(0.007)$ & $(0.004)$ & $(0.011)$ \\
\hline \multirow[t]{2}{*}{$\mu$} & 2.278 & 2.314 & 2.251 & 2.300 & 2.160 \\
\hline & $(0.029)$ & $(0.038)$ & $(0.043)$ & $(0.031)$ & $(0.083)$ \\
\hline \multirow[t]{2}{*}{$\sigma$} & 0.521 & 0.534 & 0.489 & 0.509 & 0.578 \\
\hline & $(0.018)$ & $(0.024)$ & $(0.025)$ & $(0.019)$ & $(0.050)$ \\
\hline \multirow[t]{2}{*}{$\rho V_{n}(m)$} & 3.685 & 3.722 & 3.622 & 3.670 & 3.762 \\
\hline & $(0.082)$ & $(0.114)$ & $(0.129)$ & $(0.098)$ & $(0.141)$ \\
\hline \multirow[t]{2}{*}{$\alpha$} & 0.378 & 0.387 & 0.369 & 0.382 & 0.357 \\
\hline & $(0.008)$ & $(0.005)$ & $(0.014)$ & $(0.005)$ & $(0.008)$ \\
\hline$N$ & 1781 & 931 & 850 & 1423 & 358 \\
\hline \multirow[t]{2}{*}{$\ln L$} & -4329.615 & -2354.074 & -1958.596 & -3399.592 & -911.163 \\
\hline & \multicolumn{5}{|c|}{ Including All $w<m$} \\
\hline \multirow[t]{2}{*}{$\lambda$} & 0.312 & 0.269 & 0.379 & 0.332 & 0.291 \\
\hline & $(0.022)$ & $(0.026)$ & $(0.039)$ & $(0.029)$ & $(0.038)$ \\
\hline \multirow[t]{2}{*}{$\eta$} & 0.038 & 0.035 & 0.044 & 0.033 & 0.063 \\
\hline & $(0.004)$ & $(0.005)$ & $(0.006)$ & $(0.004)$ & $(0.011)$ \\
\hline \multirow[t]{2}{*}{$\mu$} & 2.282 & 2.319 & 2.253 & 2.305 & 2.160 \\
\hline & $(0.036)$ & $(0.044)$ & $(0.059)$ & $(0.041)$ & $(0.089)$ \\
\hline \multirow[t]{2}{*}{$\sigma$} & 0.493 & 0.516 & 0.454 & 0.476 & 0.569 \\
\hline & $(0.019)$ & $(0.026)$ & $(0.028)$ & $(0.021)$ & $(0.051)$ \\
\hline \multirow[t]{2}{*}{$\rho V_{n}(m)$} & 3.174 & 3.420 & 2.895 & 3.019 & 3.615 \\
\hline & $(0.148)$ & $(0.167)$ & $(0.271)$ & $(0.195)$ & $(0.175)$ \\
\hline \multirow[t]{2}{*}{$\alpha$} & 0.409 & 0.405 & 0.415 & 0.421 & 0.366 \\
\hline & $(0.006)$ & $(0.006)$ & $(0.012)$ & $(0.009)$ & $(0.021)$ \\
\hline$N$ & 1859 & 952 & 907 & 1494 & 365 \\
\hline $\ln L$ & -4535.684 & -2415.759 & -2095.628 & -3588.165 & -929.367 \\
\hline
\end{tabular}


Table 4

Model Estimates with Profit Information

September and October 1996

\begin{tabular}{ccccc} 
& \multicolumn{5}{c}{ Sample and Specification } \\
Parameter & $S 1$ & $S 1$ & $S 2$ & $S 2$ \\
$\lambda$ & & & & \\
& 0.309 & 0.314 & 0.306 & 0.307 \\
$\eta$ & $(0.015)$ & $(0.016)$ & $(0.015)$ & $(0.017)$ \\
& 0.039 & 0.039 & 0.036 & 0.036 \\
$\mu$ & $(0.003)$ & $(0.003)$ & $(0.003)$ & $(0.003)$ \\
& 2.284 & 2.246 & 2.279 & 2.274 \\
$\sigma$ & $(0.020)$ & $(0.022)$ & $(0.026)$ & $(0.089)$ \\
& 0.531 & 0.549 & 0.502 & 0.503 \\
$\rho V_{n}(4.25)$ & $(0.012)$ & $(0.013)$ & $(0.014)$ & $(0.041)$ \\
& 3.850 & 3.784 & 3.273 & 3.233 \\
$\rho V_{n}(4.75)$ & $(0.049)$ & $(0.055)$ & $(0.100)$ & $(0.415)$ \\
& - & 4.188 & - & 3.345 \\
$\alpha$ & & $(0.059)$ & & $(0.327)$ \\
& $(0.370$ & 0.369 & 0.403 & 0.405 \\
& & $(0.003)$ & $(0.004)$ & $(0.015)$ \\
$N$ & 3543 & 3543 & 3790 & 3790 \\
& & & & \\
$\ln L$ & -8664.423 & -8644.712 & -9226.423 & -9225.673
\end{tabular}


Table 5

Model Estimates Using Profit Information

September and October CPS-ORG

"Endogenous" $\lambda$

\begin{tabular}{ccccc} 
& \multicolumn{4}{c}{ Sample and Specification } \\
Parameter & $S 1$ & $S 1$ & $S 2$ & $S 2$ \\
$\lambda(m=4.25)$ & 0.295 & 0.299 & 0.292 & 0.292 \\
& $(0.015)$ & $(0.015)$ & $(0.015)$ & $(0.015)$ \\
$\lambda(m=4.75)$ & 0.121 & 0.125 & 0.126 & 0.126 \\
& $(0.026)$ & $(0.027)$ & $(0.026)$ & $(0.027)$ \\
$\eta$ & 0.034 & 0.034 & 0.032 & 0.032 \\
& $(0.003)$ & $(0.003)$ & $(0.003)$ & $(0.003)$ \\
$\mu$ & 2.288 & 2.250 & 2.284 & 2.279 \\
$\sigma$ & $(0.020)$ & $(0.022)$ & $(0.021)$ & $(0.028)$ \\
& 0.528 & 0.545 & 0.497 & 0.497 \\
$\rho V_{n}(4.25)$ & $(0.012)$ & $(0.013)$ & $(0.012)$ & $(0.014)$ \\
& 3.841 & 3.774 & 3.236 & 3.197 \\
$\rho V_{n}(4.75)$ & $(0.051)$ & $(0.056)$ & $(0.080)$ & $(0.116)$ \\
& - & 4.177 & - & 3.306 \\
$\alpha$ & & $(0.061)$ & & $(0.122)$ \\
& 0.370 & 0.370 & 0.406 & 0.408 \\
& $(0.006)$ & $(0.003)$ & $(0.003)$ & $(0.005)$ \\
$N$ & & & & \\
& 3543 & 3543 & 3790 & 3790 \\
$\ln L$ & -8654.609 & -8635.396 & -9217.555 & -9216.887
\end{tabular}


Table 6

Optimal Minimum Wages

Entire Sample

(Uses Estimates from Table 3, See Text)

\begin{tabular}{ccccc} 
& & \multicolumn{3}{c}{ Criteria } \\
\cline { 3 - 5 } Characteristics & Baseline & $V_{n}(m)$ & "Median Voter" & $E_{S S} S(\theta \mid m)$ \\
Optimal $m$ & & & & \\
Percent Improvement & 4.25 & 8.46 & 10.90 & 9.02 \\
Unemployment Rate & 0.113 & 0.165 & 0.227 & 0.177
\end{tabular}




\section{References}

[1] Cahuc, P., Postel-Vinay, F. and J-M Robin. "Wage Bargaining with On-the-Job Search: A Structural Econometric Model." Mimeo, University of Paris I, 2002.

[2] Card, D. and A. Krueger. Myth and Measurement: The New Economics of the Minimum Wage. Princeton: Princeton University Press, 1995.

[3] Del Boca, D. and C. Flinn. "The Determination of Welfare in Nonintact Families," with D. Del Boca. Chapter 5 in The Measurement of Household Welfare (Blundell, Preston, and Walker, eds.). Cambridge: Cambridge University Press, 1994.

[4] Dey, M. and C. Flinn. "An Equilibrium Model of Health Insurance Provision and Wage Determination." Mimeo, New York University, February 2003.

[5] Dickens, R., S. Machin, and A. Manning. "Estimating the Effect of Minimum Wages on Employment from the Distribution of Wages: A Critical View." Mimeo, Centre for Economic Performance, London School of Economics, January 1997.

[6] Drazen, A. "Optimum Minimum Wage Legislation." Economic Journal 96 (1986): 774-784.

[7] Eckstein, Z. and K. Wolpin. "Estimating a Market Equilibrium Search Model from Panel Data on Individuals." Econometrica 58 (1990): 783-808.

[8] Eckstein, Z. and K. Wolpin. "Estimating the Effect of Labor Market Discrimination on BlackWhite Differences in Wage Offers Using a Search-Matching-Bargaining Model." Review of Economic Studies (1993).

[9] Flinn, C. "Wage and Job Mobility of Young Workers." Journal of Political Economy 94 (1986): S88-S110.

[10] Flinn, C. "Equilibrium Wage and Dismissal Processes." Journal of Business and Economic Statistics 15 (1997): 221-236 .

[11] Flinn, C. "Interpreting Minimum Wage Effects on Wage Distributions: A Cautionary Tale." Annales d'Economie et de Statistique (2003), forthcoming.

[12] Flinn, C. and J. Heckman. "New Methods for Analyzing Structural Models of Labor Force Dynamics." Journal of Econometrics 18 (1982): 115-68.

[13] Heckman, J. and G. Sedlacek. "The Impact of the Minimum Wage on the Employment and Earnings of Workers in South Carolina." In Report of the Minimum Wage Study Commission, vol. 5. Washington, D.C.: U.S. Government Printing Office, 1981.

[14] Kapteyn, A. and P. Kooreman. "On the Empirical Implementation of Some Game-Theoretic Models of Household Labor Supply." Journal of Human Resources 25 (1990): 584-598.

[15] Lang, K. "Pareto Improving Minimum Wage Laws." Economic Inquiry 25 (1987): 145-158.

[16] Manning, A. "How Do We Know That Real Wages Are Too High?" Quarterly Journal of Economics 110 (1995): 1111-1125. 
[17] Mas-Colell, A., Whinston, M. and J. Green. Microeconomic Theory. New York: Oxford University Press, 1995.

[18] Meyer, R. and D. Wise. "Discontinuous Distributions and Missing Persons: The Minimum Wage and Unemployed Youth." Econometrica 51 (1983a): 1677-1698.

[19] Meyer, R. and D. Wise. "The Effects of the Minimum Wage on Employment and Earnings of Youth." Journal of Labor Economics 1 (1983b): 66-100.

[20] Neumark, D. and W. Wascher. "Employment Effects of Minimum Wage and Subminimum Wages: Panel Data on State Minimum Wage Laws." Industrial and Labor Relations Review 46 (1992): 55-81.

[21] Neumark, D. and W. Wascher. "Minimum-Wage Effects on School and Work Transitions of Teenagers." American Economic Review 85 (1995): 244-249.

[22] Postel-Vinay, F. and J-M Robin. "Equilibrium Wage Dispersion with Heterogeneous Workers and Firms." Econometrica 70 (2002): 2295-1350.

[23] Rebitzer, J. and L. Taylor. "The Consequences of Minimum Wage Laws: Some New Theoretical Ideas." Journal of Public Economics 56 (1996): 245-256.

[24] Rubinstein, A. "Perfect Equilibrium in a Bargaining Model." Econometrica 50 (1982): 97-110.

[25] Swinnerton, K. "Minimum Wages in an Equilibrium Search Model with Diminishing Returns to Labor in Production." Journal of Labor Economics 14 (1996): 340-355.

[26] Yashiv, Eran. "Bargaining, the Value of Unemployment, and the Behavior of Aggregate Wages." Mimeo, Tel Aviv University, 2003. 
Figure 1.a

Hourly Wages

September 1996

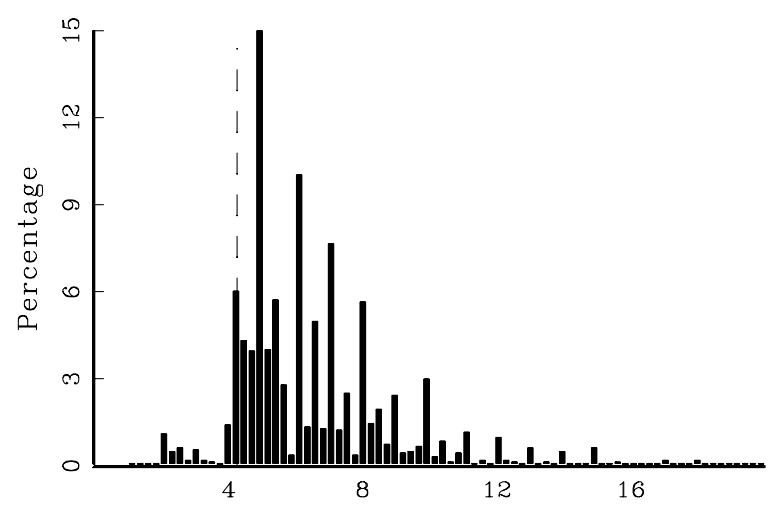

w

Figure 1.c

Hourly Wages

October 1996

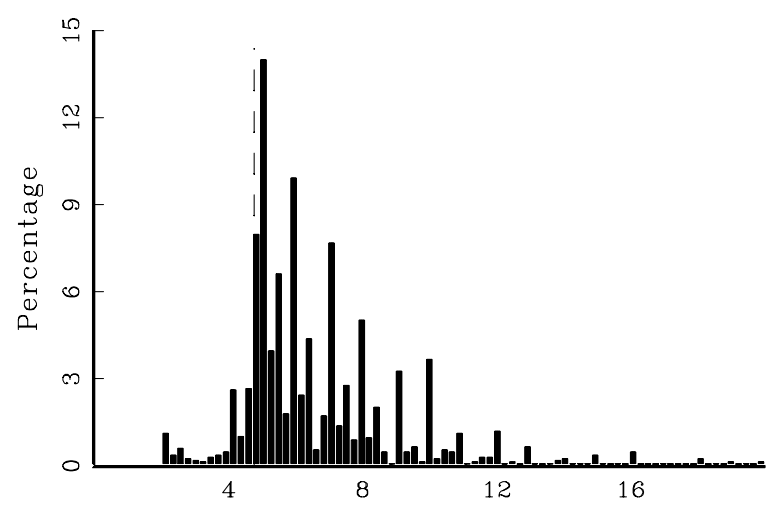

Figure 1.b

Unemployment Durations

September 1996

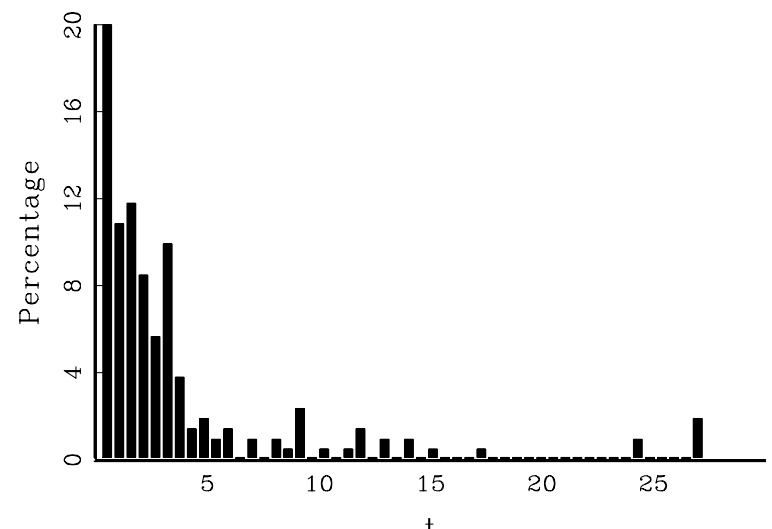

Figure 1.d

Unemployment Durations

October 1996

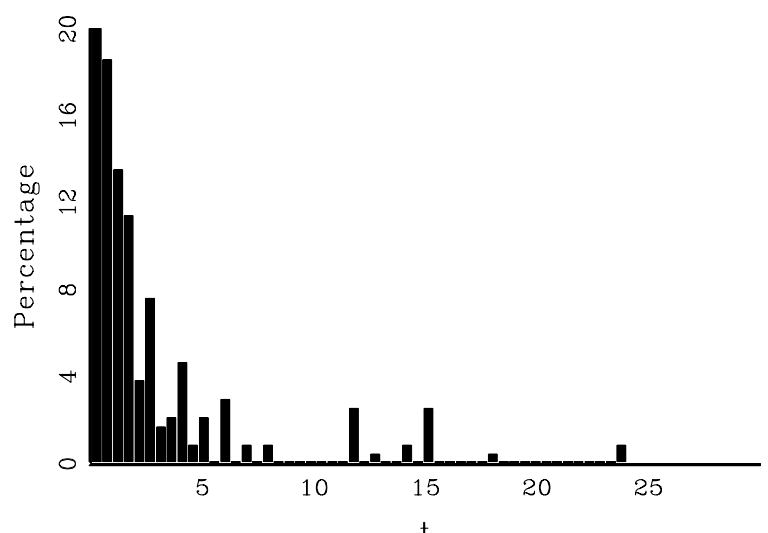


Figure 2.a

Match Value Density

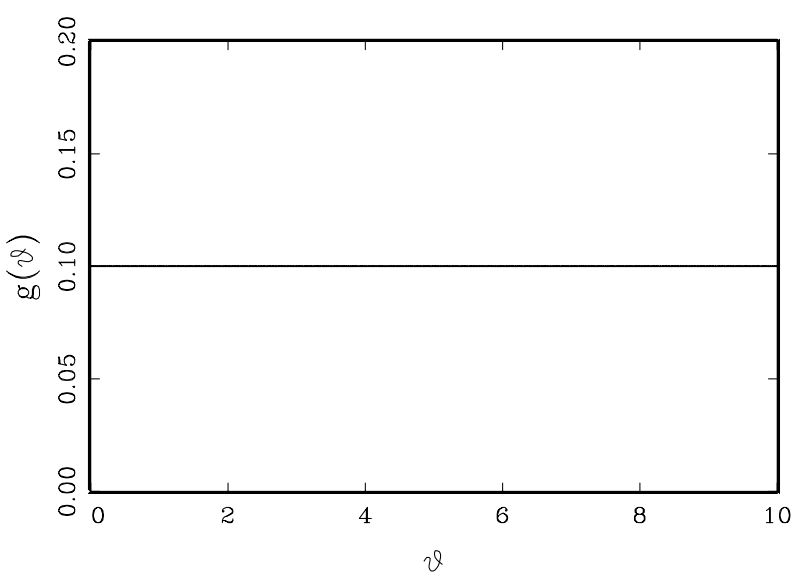

Figure 2.c Wage Density $\alpha=.30$

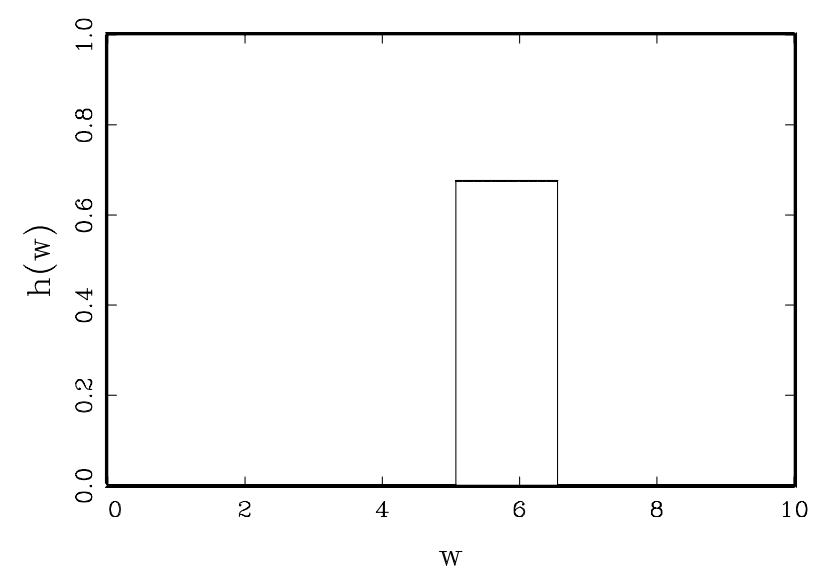

Figure 2.b

Equilibrium Wage Function

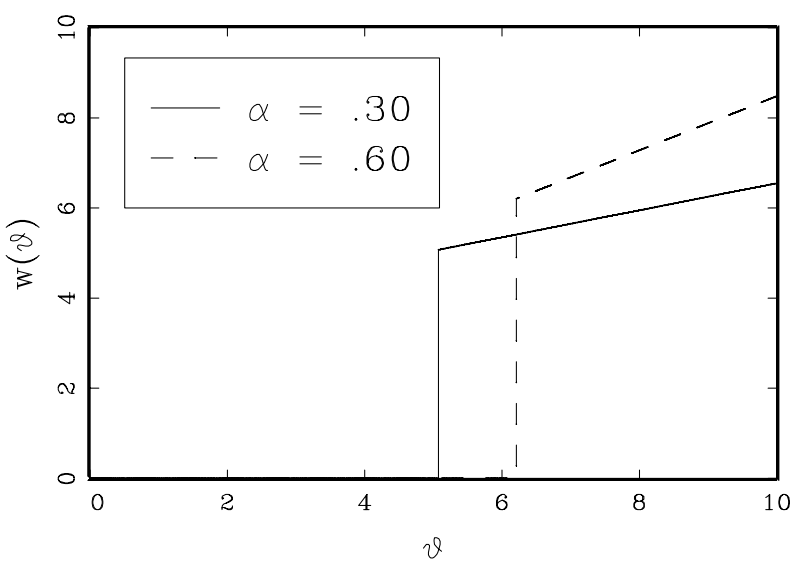

Figure 2.d Wage Density

$\alpha=.60$

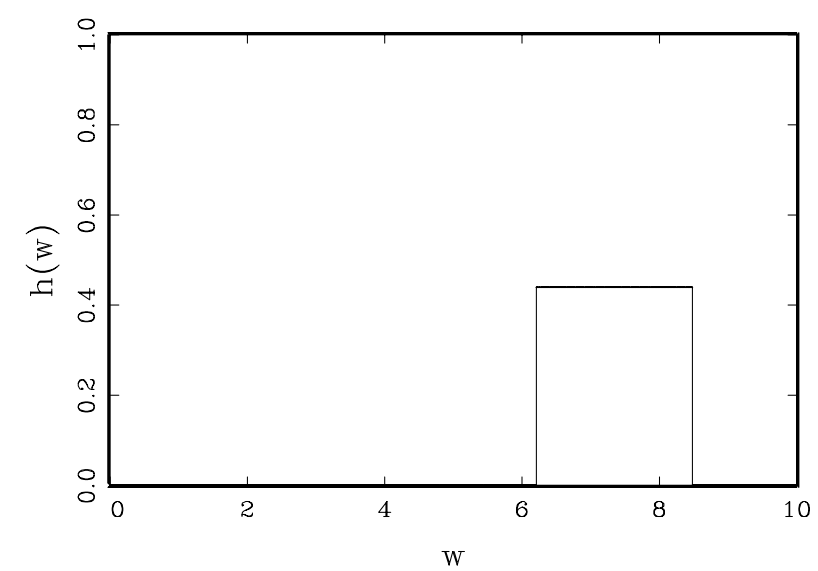


Figure 3.a

Match Value C.D.F.

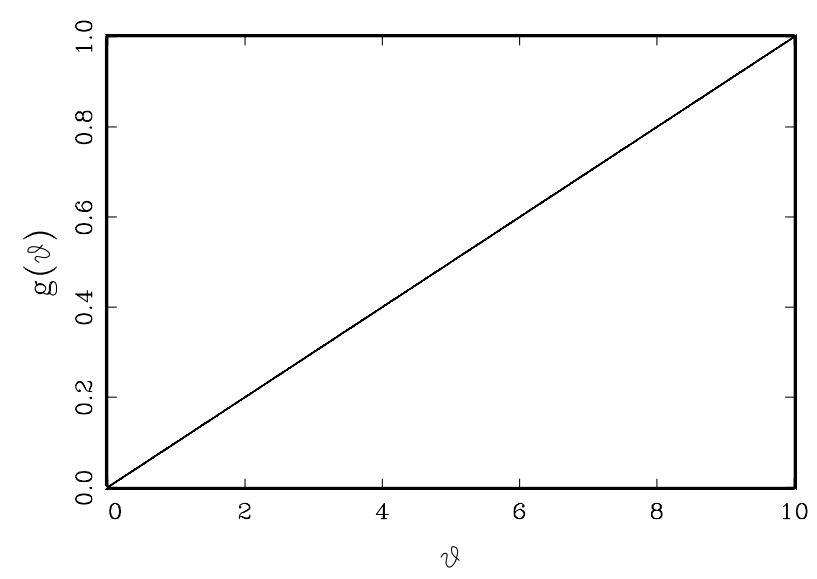

Figure 3.c Wage C.D.F $\alpha=.30$

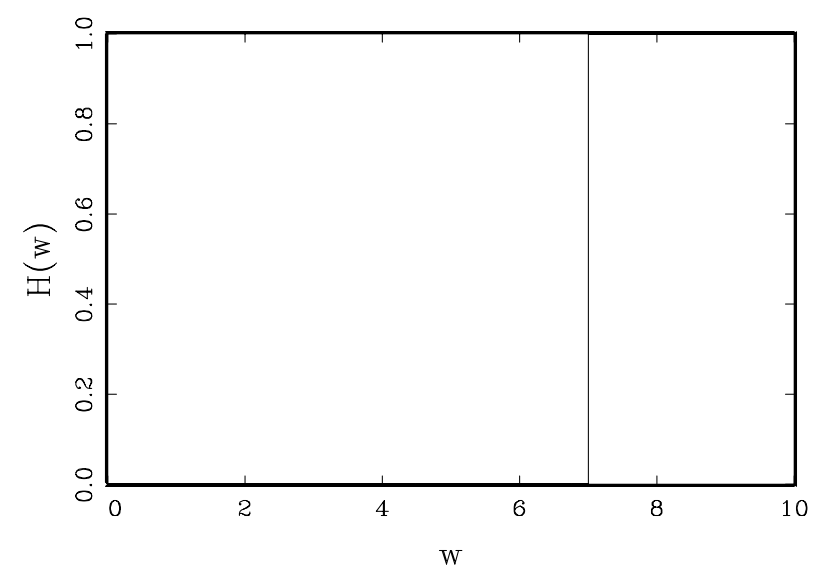

Figure 3.b

Equilibrium Wage Functions

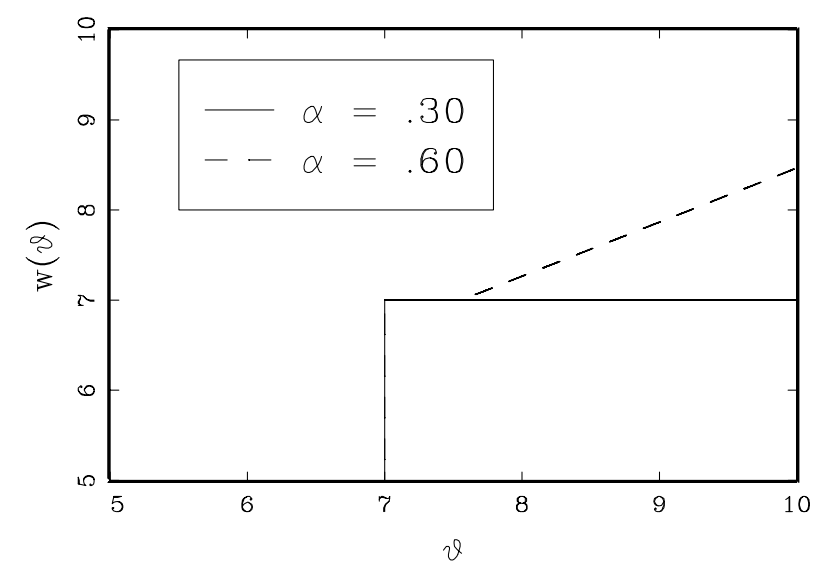

Figure 3.d Wage C.D.F $\alpha=.60$

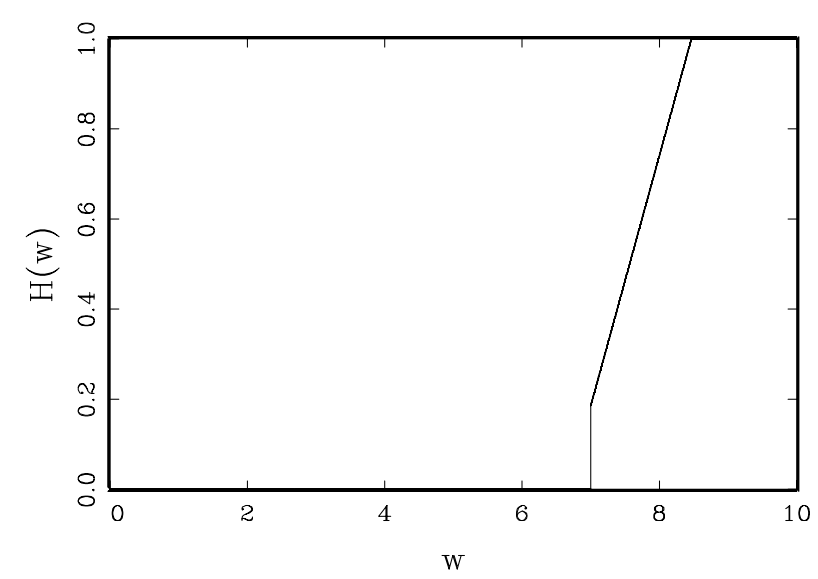


Figure 4.a

$\mathrm{V}_{\mathrm{n}}(\mathrm{m})$

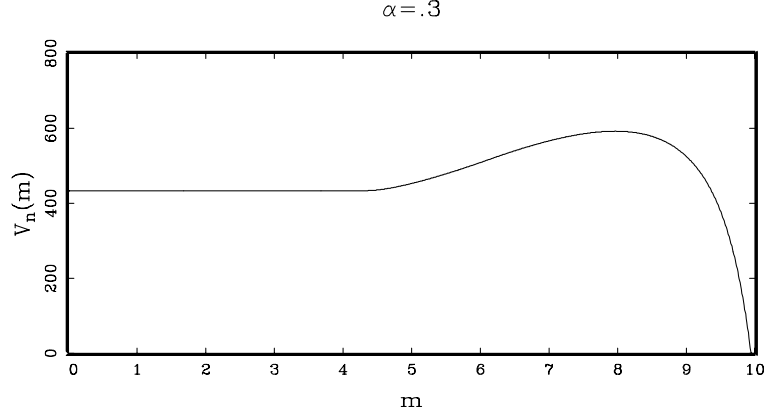

Figure 4.0

$\mathrm{S}(\vartheta \mid \mathrm{m})$

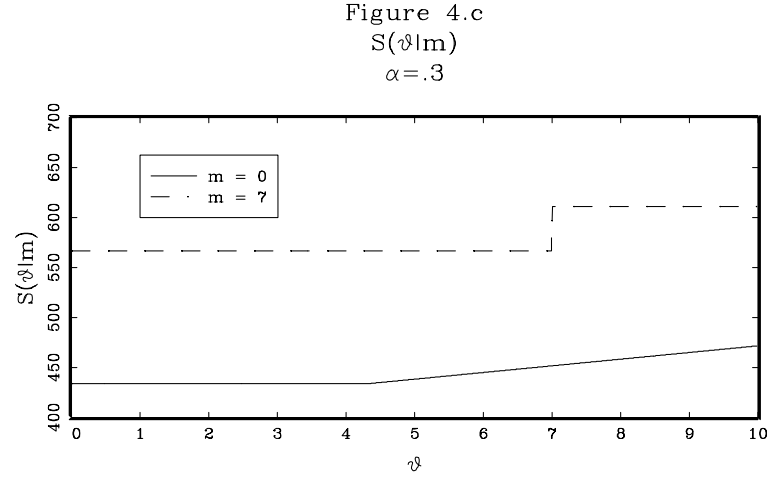

Figure 4.e

$\mathrm{E}_{\mathrm{SS}} \mathrm{S}(\vartheta \mid \mathrm{m})$

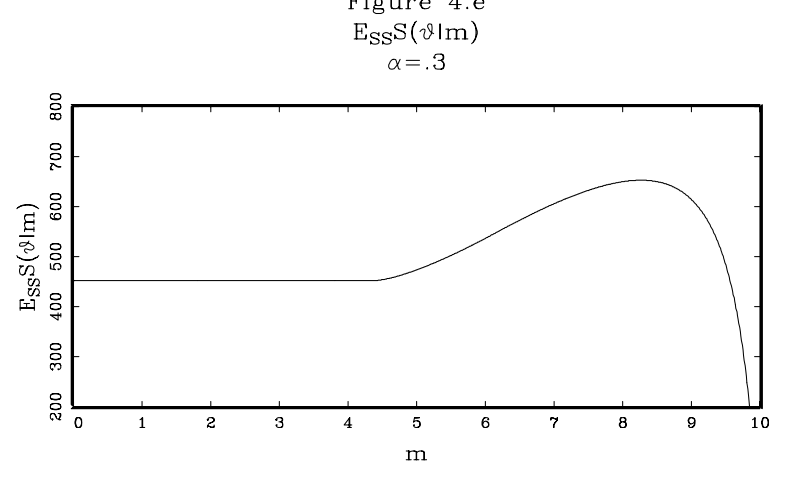

Figure 4.b

$\mathrm{V}_{\mathrm{n}}(\mathrm{m})$

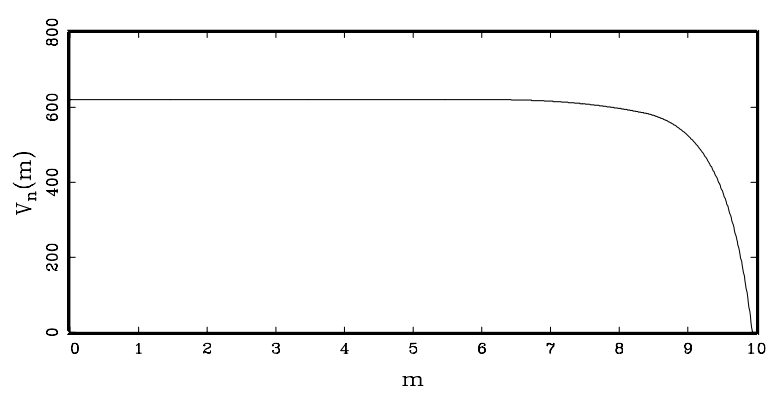

Figure 4.

$\mathrm{S}(\vartheta \mid \mathrm{m})$

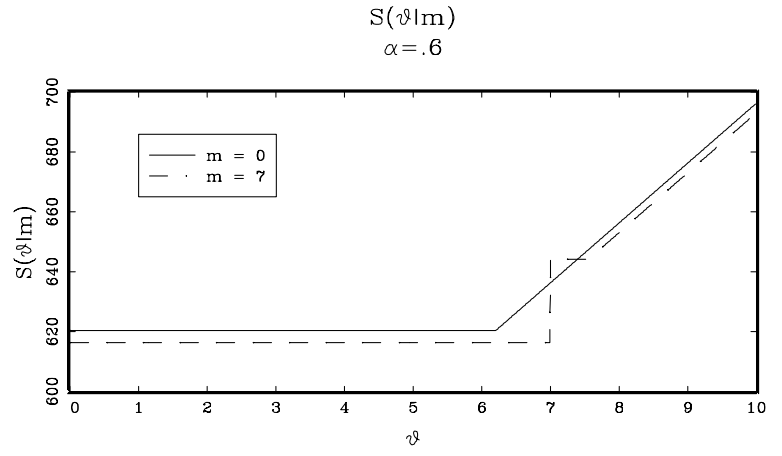

Figure 4.

$\mathrm{E}_{\mathrm{SS}} \mathrm{S}(\vartheta \mid \mathrm{m})$

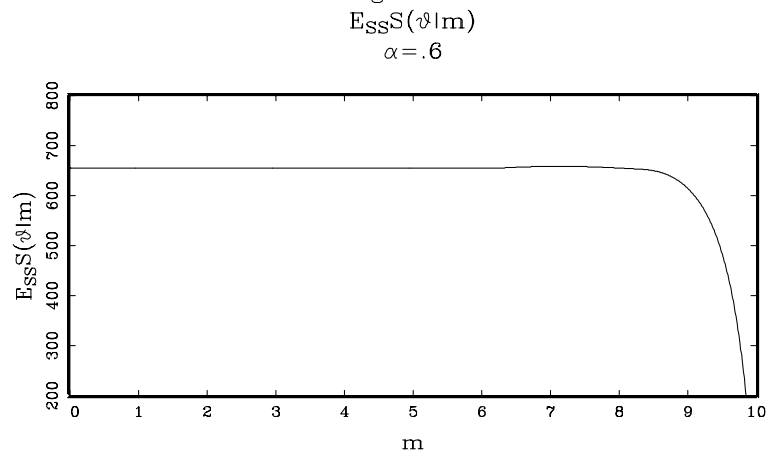




$$
J_{n} H_{i} \nabla^{\prime}
$$



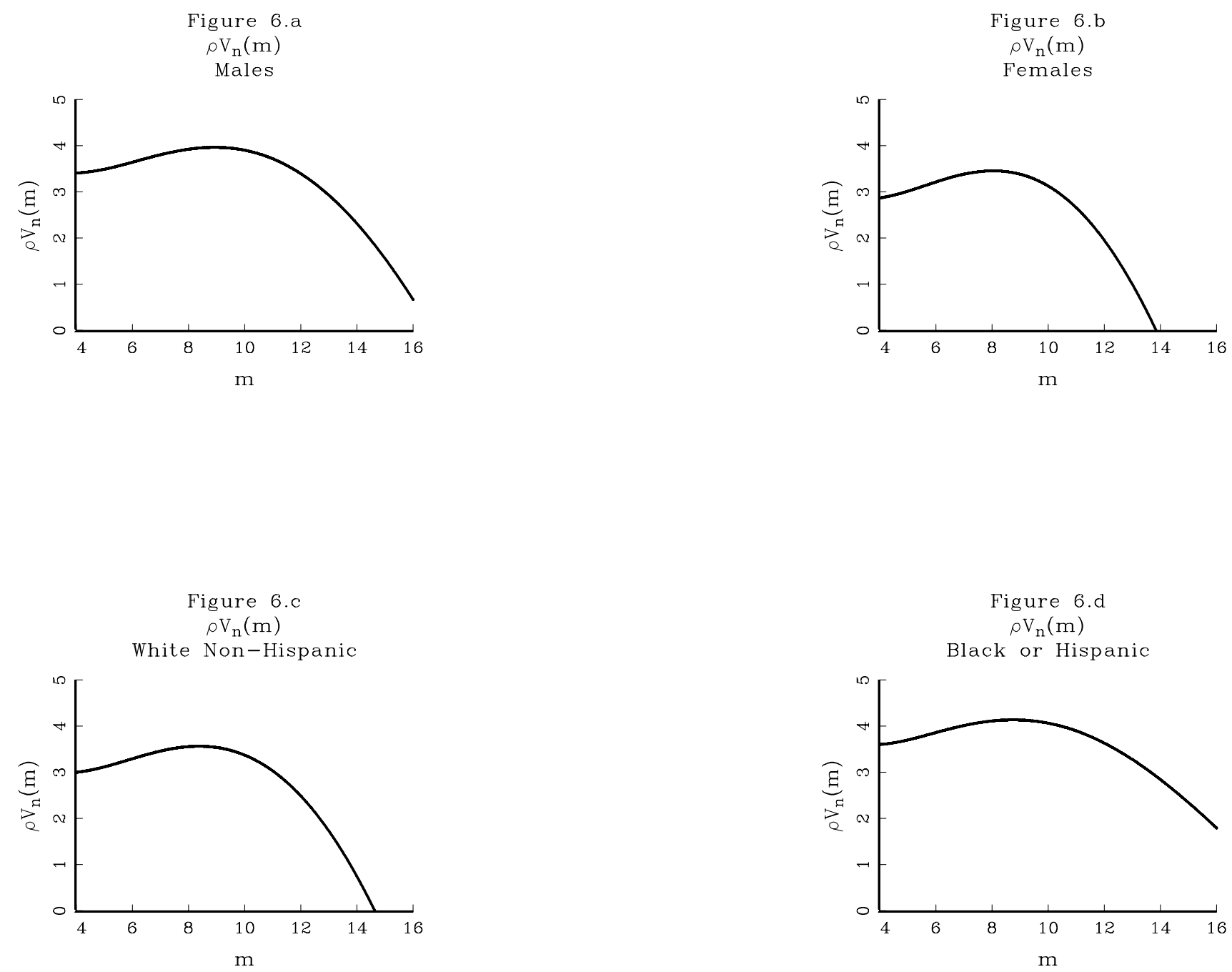
Figure 7.a

Proportion with Welfare Improvement Males

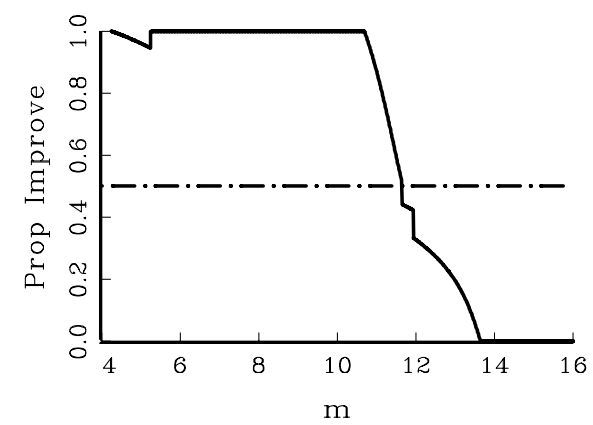

Figure 7.c

Proportion with Welfare Improvement White Non-Hispanic

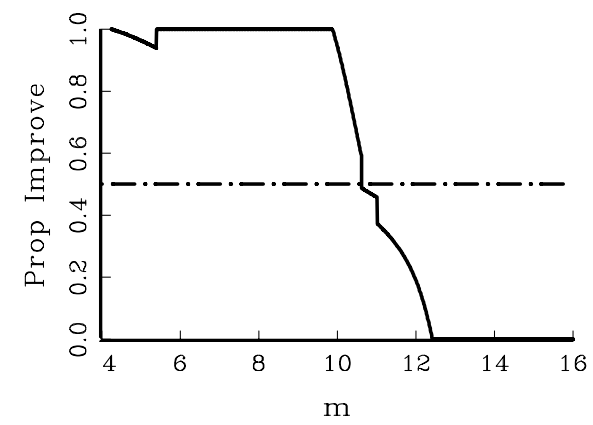

Figure 7.b

Proportion with Welfare Improvement Females

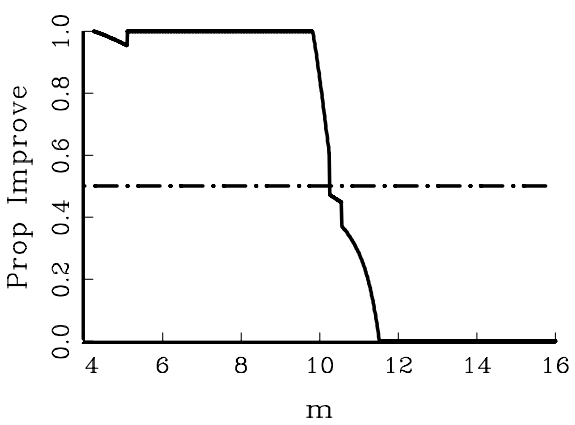

Figure 7.d

Proportion with Welfare Improvement Blacks and Hispanics

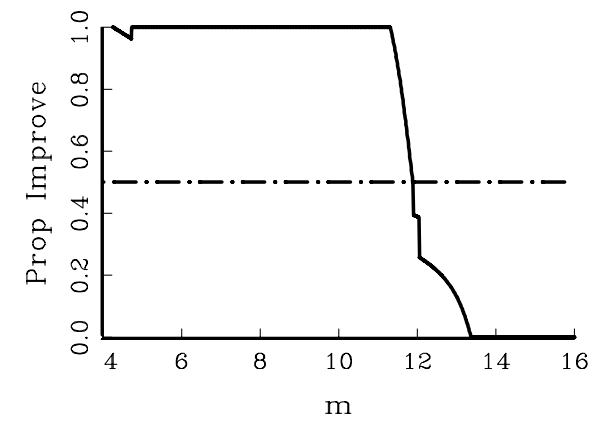



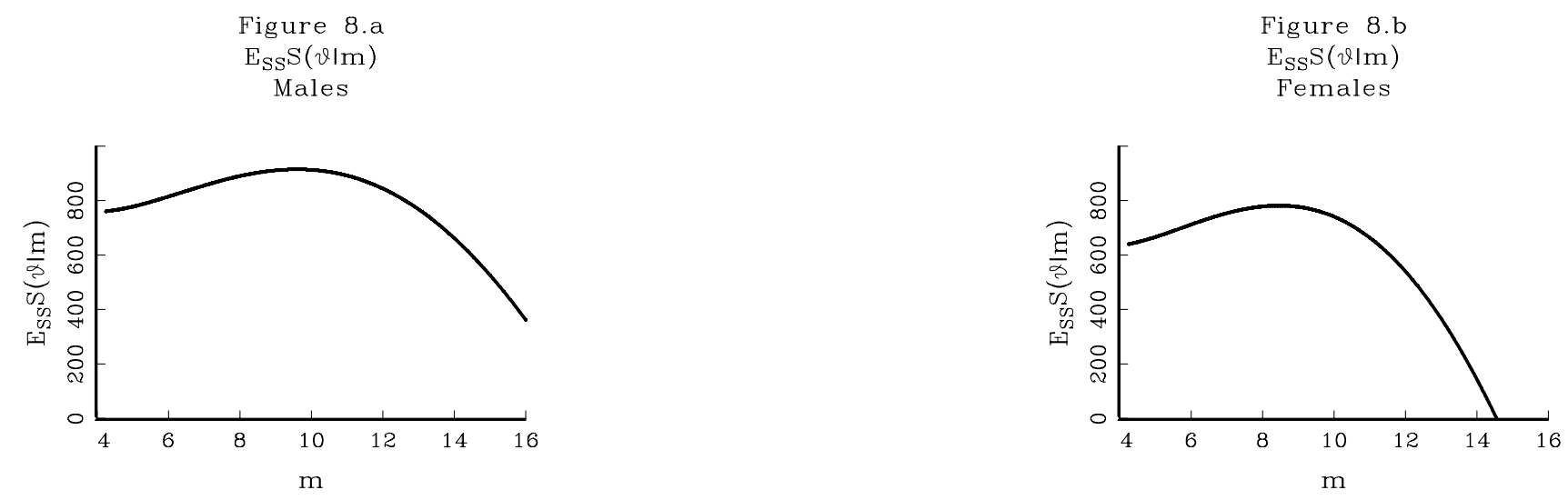

Figure 8.c

$\mathrm{E}_{\mathrm{SS}} \mathrm{S}(\vartheta \mid \mathrm{m})$

White Non-Hispanic

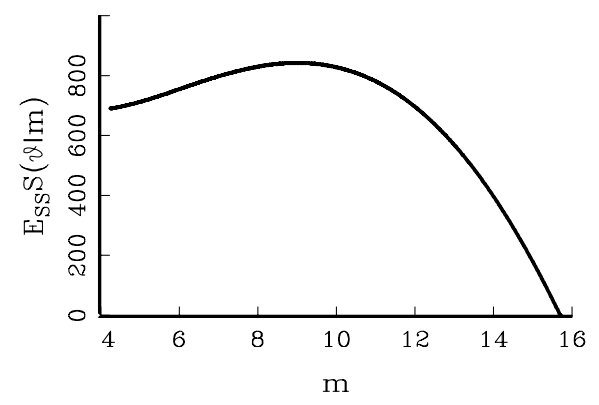

Figure 8.d

$\mathrm{E}_{\mathrm{SS}} \mathrm{S}(\vartheta / \mathrm{m})$

Blacks and Hispanics

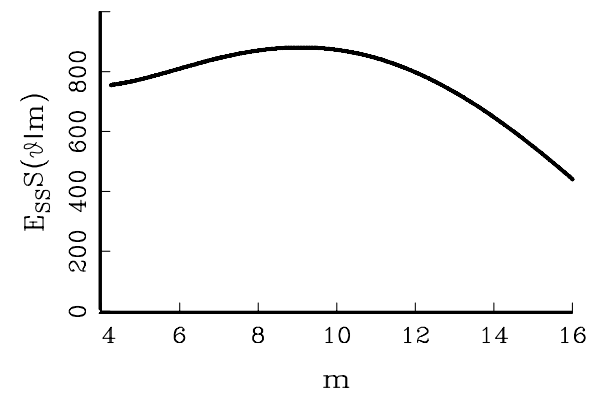




\section{IZA Discussion Papers}

\begin{tabular}{|c|c|c|c|c|}
\hline No. & Author(s) & Title & Area & Date \\
\hline 935 & T. J. Dohmen & $\begin{array}{l}\text { Performance, Seniority and Wages: Formal } \\
\text { Salary Systems and Individual Earnings Profiles }\end{array}$ & 1 & $11 / 03$ \\
\hline 936 & $\begin{array}{l}\text { M. K. Jones } \\
\text { P. L. Latreille } \\
\text { P. J. Sloane }\end{array}$ & Disability, Gender and the Labour Market & 3 & $11 / 03$ \\
\hline 937 & $\begin{array}{l}\text { W. Eggert } \\
\text { L. Goerke }\end{array}$ & $\begin{array}{l}\text { Fiscal Policy, Economic Integration and } \\
\text { Unemployment }\end{array}$ & 2 & $11 / 03$ \\
\hline 938 & $\begin{array}{l}\text { D. Del Boca } \\
\text { A. Venturini }\end{array}$ & Italian Migration & 1 & $11 / 03$ \\
\hline 939 & E. Toulemonde & $\begin{array}{l}\text { Acquisition of Skills, Education Subsidies, and } \\
\text { Agglomeration of Firms }\end{array}$ & 2 & $11 / 03$ \\
\hline 940 & $\begin{array}{l}\text { A. Constant } \\
\text { Y. Shachmurove } \\
\text { K. F. Zimmermann }\end{array}$ & $\begin{array}{l}\text { What Makes an Entrepreneur and Does It Pay? } \\
\text { Native Men, Turks, and Other Migrants in } \\
\text { Germany }\end{array}$ & 1 & $11 / 03$ \\
\hline 941 & $\begin{array}{l}\text { R. V. Burkhauser } \\
\text { J. S. Butler } \\
\text { G. Gumus }\end{array}$ & $\begin{array}{l}\text { Option Value and Dynamic Programming Model } \\
\text { Estimates of Social Security Disability Insurance } \\
\text { Application Timing }\end{array}$ & 6 & $11 / 03$ \\
\hline 942 & $\begin{array}{l}\text { R. V. Burkhauser } \\
\text { J. S. Butler } \\
\text { G. Gumus }\end{array}$ & $\begin{array}{l}\text { Dynamic Modeling of the SSDI Application } \\
\text { Timing Decision: The Importance of Policy } \\
\text { Variables }\end{array}$ & 6 & $11 / 03$ \\
\hline 943 & $\begin{array}{l}\text { J. T. Addison } \\
\text { P. Teixeira }\end{array}$ & $\begin{array}{l}\text { What Have We Learned About the Employment } \\
\text { Effects of Severance Pay? Further Iterations of } \\
\text { Lazear et al. }\end{array}$ & 3 & $11 / 03$ \\
\hline 944 & $\begin{array}{l}\text { H. Görg } \\
\text { D. Greenaway }\end{array}$ & $\begin{array}{l}\text { Much Ado About Nothing? Do Domestic Firms } \\
\text { Really Benefit from Foreign Direct Investment? }\end{array}$ & 2 & $11 / 03$ \\
\hline 945 & $\begin{array}{l}\text { R. Schöb } \\
\text { D. E. Wildasin }\end{array}$ & $\begin{array}{l}\text { Economic Integration and Labor Market } \\
\text { Institutions: Worker Mobility, Earnings Risk, and } \\
\text { Contract Structure }\end{array}$ & 2 & $12 / 03$ \\
\hline 946 & M. Leonardi & $\begin{array}{l}\text { Earnings Instability of Job Stayers and Job } \\
\text { Changers }\end{array}$ & 1 & $12 / 03$ \\
\hline 947 & U. Sunde & $\begin{array}{l}\text { Potential, Prizes and Performance: Testing } \\
\text { Tournament Theory with Professional Tennis } \\
\text { Data }\end{array}$ & 7 & $12 / 03$ \\
\hline 948 & $\begin{array}{l}\text { A. Kugler } \\
\text { G. Pica }\end{array}$ & $\begin{array}{l}\text { Effects of Employment Protection and Product } \\
\text { Market Regulations on the Italian Labor Market }\end{array}$ & 6 & $12 / 03$ \\
\hline 949 & C. J. Flinn & $\begin{array}{l}\text { Minimum Wage Effects on Labor Market } \\
\text { Outcomes under Search with Bargaining }\end{array}$ & 6 & $12 / 03$ \\
\hline
\end{tabular}

An updated list of IZA Discussion Papers is available on the center's homepage www.iza.org. 